\title{
VII.
}

Aus dem pharmakologischen Institnt zu Halle a. S.

\section{Die chemische Konstitution des Morphins in ihrer Beziehung zur Wirkung.}

Zweite Abhandling.

$$
\text { Von }
$$

Dr. med. Ernst Vahlen,

Privatdocent und Assistent des Institutes.

In meiner ersten in diesem Archiv ${ }^{1)}$ unter dem Titel: "Die ehemische Konstitution des Morphins in ihrer Beziehung zur Wirkung" veröffentlichten Arbeit waren nach jahrelangen Bemühungen Ergebnisse mitgeteilt worden, die nur dureh unausgesetztes Ineinandergreifen von chemischer Synthese und pharmakologischem Tierexperiment gewonnen werden konnten.

In jener Mitteilung war, entgegen der bis dahin geltenden Meinung, die niemals Widerspruch, sondern sogar Aufnahme in chemische und pharmakologische Lehrbücher gefunden hatte, daf nämlich der physiologiseh wirksame Teil des Morphinmoleküles der Morpholinring sei, der Beweis geliefert, daß diese Rolle vielmehr dem Phenanthrenring zukommt, was vordem, soweit mir bekannt, nicht einmal vermutungsweise geäußert worden war.

Ein wesentlicher Teil meiner Beweisführung knüpfte sich an das Studium eines ron mir Epiosin genannten Phenanthrenderivates, von dem ich gezeigt habe, daß es mit dem N-Methyldiphenylenimidazol:

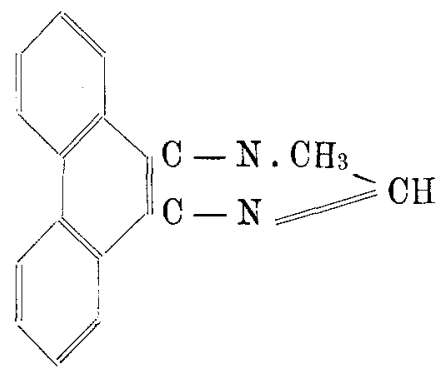

1) Dieses Archiv. 47. (1902.) 365. 
identisch ist. Dieses Epiosin Erystallisiert aus Alkohol in glashellen Prismen vom Schmelzpunkt 195 und kann mit Leichtigkeit rein dargestellt werden. $\mathrm{Zu}$ allen meinen Tierversuchen verwandte ich das in Nadeln krystallisierende, in Wasser leicht lösliche Chlorid des Epiosins. Der Reinheit meiner Präparate, dies sei hier ausdrücklich hervorgeboben, habe ich mich stets vergew is sert.

Das Epiosin erwies sich nun in seiner Wirkung auf Frösche wie auf Säugetiere (Hunde), als ein Narkotikum, welches das Zentralnervensystem in ähnlicher Weise ergriff, wie die Alkaloide der Morphingruppe. Daß ich mich bei meinen Versuchen in erster Linie des Hundes bediente, latte seinen guten Grund; denn der Hund, wenngleich außerordentlich viel weniger leicht als der Mensch und fast mit noch größeren individuellen Unterschieden als dieser auf die narkotische Wirkung der Opinmalkaloide reagierend, ist doch nach allen bisherigen Erfahrungen dasjenige Tier, das weitaus am empfindlichsten gegen die Wirkungen des Morphins ist. Ja, ich glaube kaum zu viel zu sagen, wenn ich behaupte, daß der Hund für das Studium morphiumähnlicher Wirkungen, namentlich von Substanzen, bei denen diese Eigenschaft erst entdeckt werden soll, das einzig brauchbare Versuchstier darstellt. Die Verwendbarkeit von Kaninchen in dieser Hinsicht ist schon sehr viel geringer; denn die Empfindlichkeit des Kaninchens für die narkotisehe Wirkung des Morphins ist erheblich schwächer als die des Hundes. Zum Studium morphiumähnlicher Wirkungen aber andere Warmblüter als Hunde und Kaninchen zu benützen, halte ich geradezu für einen pharmakologischen Kunstfehler.

In meiner ersten Arbeit war auch der Einflub des Epiosins auf Atmung und Blutdruck bereits Gegenstand einiger Versuche gewesen, aus denen geschlossen wurde, daß durch Epiosin die Atmung verlangsamt und abgeflacht, der Blutdruck erhöht wird. Es schien mir aber wïnschenswert, diese beiden Epiosinwirkungen noeh in genauen Maßzahlen zur Anschauung zu bringen.

I. Die Wirkung des Epiosins auf die Atmung.

$\mathrm{Zu}$ diesen Experimenten wurden auch Kaninchen angewandt Es seien daher zwei einfache Vergiftungsversuche, welche die Wirkung des Epiosins auf diese Tiere in ibren Grundzïgen erkennen lassen, vorangeschickt. 


\section{Versuch Nr. I.}

Graues Kaninchen, $1600 \mathrm{~g}$, sehr lebhaft und empfindlich.

$5 \mathrm{~h} .55 \mathrm{~m}$. Intravenöse Injektion von $1 \mathrm{ccm}$ einer 10 proz. Epiosinchloridlösung $(=0,1$ Epiosinchlorid) in die linke Jugularvene. Dauer der Injektion knapp eine Minate.

Noch bevor die Injektion vollendet ist, liegt das Tier bereits regungslos da. Von den Fesseln befreit, mit denen es auf dem Brett befestigt war, bleibt es ruhig liegen und führt keine spontanen Bewegungen aus. Pupillen nicht verengt. Kornealreflex nicht erloschen, wohl aber herabgesetzt. Es wird die Kanüle herausgenommen und die Wunde zugenäht. Dabei ist das Tier nicht gefesselt, bleibt aber trotzdem völlig ruhig liegen, ohne beim Nähen auch nur im geringsten zu zucken. Man kann es auf eine Seite legen, es bleibt liegen. Nach einiger Zeit dreht es sich um, so daß es auf dem Bauch liegt, den Kopf auf die Tischplatte gestützt. Es bewegt sich spontan nicht von der Stelle. Man kann es in die Ohren kneifen oder auf die Pfoten schlagen, es erfolgt keine Reaktion.

$6 \mathrm{~h} .30 \mathrm{~m}$. Liegt mit halbgeschlossenen Augen auf dem Bauch. In die Ohren oder den Schwanz gekniffen oder auf die Pfoten geschlagen, reagiert es nicht.

Dieser deutlich narkotische Zustand dauert, allmählich abnehmend, etwa bis $7 \mathrm{~h} .30 \mathrm{~m}$.

\section{Versuch Nr. II.}

Dasselbe Tier wie zu Versuch $\mathrm{Nr}$. I.

$4 \mathrm{~h} .50 \mathrm{~m}$. Injektion von $2 \mathrm{ccm}$ einer 10 proz. Epiosinchloridlösung (=0,2 Epiosinchlorid) in die Karotis in peripherer Richtung. Während der Injektion und zwar gleich zu Beginn, zeigt sich das Tier sehr erregt. Am Schluß der etwa eine Minute dauernden Injektion liegt das Tier reaktionslos da. Kornealreflex vollkommen erloschen. Papillen nicht verengt. Das Tier wird anf die Seite gelegt, es bleibt liegen. Alle Reflexe sind erloschen. Atmung ruhig. Herzschlag etwas verlangsamt.

$5 \mathrm{~h} .10 \mathrm{~m}$. Die Atmung wird aussetzend.

5 h. $18 \mathrm{~m}$. Der Tod tritt ohne vorherige Krämpfe ein.

Die sofort ausgeführte Sektion ergiebt keine akuten Organveränderungen. Beide Herzventrikel sind mit flüssigem Blute gefüllt, dessen Farbe nicht den geringsten Verdacht auf Anwesenheit von Methämoglobin hätte erwecken können. Auch bei spektroskopischer Untersuchung einer Blutprobe läßt sich der charakteristische Methämoglobinstreifen nicht entdecken.

\section{Versuch Nr. III.}

Weißes männliches Kaninchen, $1010 \mathrm{~g}$ schwer. Sollte zu einem Respirationsversuch dienen und wurde deshalb tracheotomiert. Da aber schon die normale Respirationskapazität des Tieres sehr gering war, wurde darauf verzichtet die Wirkung des Epiosins auf die Atmung genau festzustellen.

$5 \mathrm{~h} .35 \mathrm{~m}$. Intravenöse Injektion von $2 \mathrm{ccm}$ einer 10 proz. Lösung von Epiosinchlorid $(=0,2$ dieses Salzes) injiziert. Dauer der Injektion etwa eine halbe Minute. 
Gleich nach der Injektion erweist sich die Empfindlichkeit, die vor dem Versuch geprüft worden war, ganz außerordentlich herabgesetzt, aber nicht vollkommen erloschen.

$5 \mathrm{~h}, 45 \mathrm{~m}$. Weitere intravenöse Injektion von noch $1 \mathrm{ccm}$ obiger Epiosinlösung.

Gleich darauf zeigt sich die Empfindlichkeit vollkommen erlosehen. Die Pfoten und die Schwanzwurzel können mittelst eines Nadelhalters zermalmt werden, ohne daß eine Zuckung oder ein Schrei erfolgt. Das Tier wird losgebunden und bleibt bis zum Schluß des Versuches ungefesselt ruhig auf dem Rücken liegen. Kornealreflex erloschen.

5 h. $47 \mathrm{~m}$. Abgang von Kot.

5 h. $50 \mathrm{~m}$. Die Knochen der Pfoten und der Sehwanzwurze können mit dem Nadelhalter zermalmt werden ohne irgend eine Reaktion von seiten Tieres. Kornealreflex erloschen. Pupillen nicht verengt.

Der Atmungstypus erinnert an das Che yne-Stokes sche Phänomen. Perioden von sehr großer und sehr geringer Atemfrequenz wechseln miteinander ab. Die Periode der verminderten Atemfrequenz schließs jedesmal mit einem auffallend tiefen Atemzuge ab.

$6 \mathrm{~h} .40 \mathrm{~m}$. Narkose noch genan so tief wie vorher.

$7 \mathrm{~h} .30 \mathrm{~m}$. Das Tier wird mit Chloroform getötet. Das unmittelbar darauf entnommene. Blut zeigt normal rote Farbe. Methämoglobin kann selbst mit dem Spektroskop darin nicht nachgewiesen werden.

Die im folgenden mitgeteilten Respirationsversuche wurden an tracheotomierten und durch Decken vor Abkiihlung geschiitzten Tieren vorgenommen, die mittelst zweier Darmventile atmeten, welche Inspirations- und Expirationsstrom voneinander trennten. Die Volumina der ausgeatmeten Luft wurden durch eine Elster sche Gasuhr gemessen. Die notierten Einzelwerte beziehen sich auf einem Zeitraum von 30 Sekunden. Gleichzeitig wurde die Atem. frequenz verzeichnet. Durch Division des Minutenatemvolumens durch die Atemfrequenz konnte für jeden Zeitabsehnitt des Versuches das Durchschnittsvolumen des einzelnen Atemzuges ermittelt werden. Die so berechneten Größen sind offenbar, trotzdem man sie in allen, zum Vergleich mit der meinigen in Betracht kommenden Arbeiten, ausgenommen derjenigen von Leichtenstern i) vermißt, ein ganz integrierender Bestandteil des Bildes, das die gesamte Atemleistung eines Tieres darbietet; denn die Volumina der einzelnen Atemzïge können bei gleichzeitiger Veränderung der Atemfrequenz um das Minutenvolum in gleichem Sinne entweder wachsen oder fallen

1) Leichtenstern, Versuche über das Volumen der unter verschiedenen Umständen ausgeatmeten Luft. Zeitschr. f. Biologie. VII. (1871.) 197. -- Vgl. anch Dreser, Über die Wirkung einiger Derivate des Morphins auf die Atmung. Pflügers Archiv. XXXII. (1898.) 493. 
oder auch unverändert bleiben. Welche von diesen drei Möglichkeiten aber im konkreten Fall in Geltung tritt, darüber können die Kurven der Frequenz und der Minutenvolumina nur einen ungefähren Aufschluß geben. Natürlich stellt die Lungenventilation, die durch die Menge der in der Zeiteinheit ein- und ausgeatmeten Luftmenge definiert ist, den wichtigsten Teil der gesamten Atemmechanik dar. Gleichwohl ist die Zahl und Beschaffenheit der einzelnen Posten, aus denen sich jene Gesamtgröße der Lungenventilation zusammensetzt, nicht unwesentlich. Denn es kann für das atmende Tier nicht einerlei sein, ob dieselbe Menge Luft in der Zeiteinheit von einer größeren Zahl oberflächlicher oder einer kleineren Zabl tiefer Atemziuge gefördert wird. Vor allen Dingen kann aber gerade die Beschaffenheit dieser einzelnen Posten einen Aufschluß über den Erregbarkeitszustand des Respirationszentrums geben. Im allgemeinen kann man bei gleichzeitiger Verminderung des in der Zeiteinheit und des mit dem einzelnen Atemzuge ausgeatmeten Luftrolumens mit großer Wahrseheinlichkeit, wenn auch nicht mit Bestimmtheit auf eine herabgesetzte Reizbarkeit des Respirationszentrums schließen. Einen sicheren Beweis kann man freilich nur mit der von $\mathrm{Zuntz}^{1}$ ) ersonnenen Methode liefern. Ich habe mich aber auf die geschilderte Versuchsanordnung beschränkt, weil sie ausreichte, um die Wirkung des Epiosins auf die Atmung mit derjenigen des Morphins in Parallele zu setzen.

$$
\text { Versuch Nr. IV. }
$$

Weißes, weibliches Kaninchen, 3390 g. Tracheotomiert. In die rechte Jugularvene eine Injektionskanüle eingebunden.

5 h. $40 \mathrm{~m}$. Operation beendigt.

5 h. $50 \mathrm{~m}$. Ausgeatmete Luft pro $1 / 2$ Minute in Kubikzentimetern:

$$
\begin{array}{lllll}
600 & 400 & 400 & 400 & 600 \\
500 & 450 & 400 & 400 & 450
\end{array}
$$

Frequenz: 62 Atemzitge pro Minute.

Volum der ausgeatmeten Luft:

in 1 Minute 900,00 in 1 Atemzug 14,51 .

$6 \mathrm{~h} .7 \mathrm{~m}$. Injektion von $1 \mathrm{ccm}$ einer 10 proz. Epiosinchloridlösung (=0,1 Epiosinchlorid)

Dauer der Injektion $1_{2}^{\prime}$ Minute.

1) Zuntz und Cohnstein, Weitere Untersuchungen zur Physiologie des Săugetierfötus. Pflügers Archiv. XLII. (1888.) 342. - Vgl. ferner Löwy, Zur Kenntnis der Erregbarkeit des Atemzentrums. Ibid. XLVII, (1890.) 601 und Winternitz, Über die Wirkung einiger Morphinderivate auf die Atmung des Menschen. Therapeut. Monatshefte. 1899. 469. 
6 h. $10 \mathrm{~m}$. Beginn der Ablesung an der Gasuhr.

$$
\begin{array}{lllll}
300 & 250 & 200 & 250 & 250 \\
250 & 300 & 300 & 250 & 250
\end{array}
$$

Frequenz: 60 Atemzüge pro Minute.

Volumen der ausgeatmeten Luft:

$6 \mathrm{~h} .18 \mathrm{~m}$.

in 1 Minute 520,00

in 1 Atemzug 8,67.

$$
\begin{array}{lll}
250 & 200 & 250 \\
200 & 250 &
\end{array}
$$

Frequenz: 61 Atemzüge pro Minute.

Volumen der ausgeatmeten Luft:

in 1 Minute 460,00 in 1 Atemzug 7,54 .

$6 \mathrm{~h} .26 \mathrm{~m}$.

$\begin{array}{llllllll}250 & 200 & 250 & 200 & 200 & 300 & 250 & 250 \\ 250 & 250 & 250 & 250 & 200 & 250 & 250 & \end{array}$

Frequenz: 64 Atemzüge pro Minute.

Volumen der ausgeatmeten Luft:

in 1 Minute 480,00 in 1 Atemzug 7,50 .

$6 \mathrm{~h} .35 \mathrm{~m}$. Kornealreflex fast ganz erloschen. Pupillen nicht verengt. Man kann mit dem Nadelhalter die Schwanzwirbel zermalmen, ohne daß das Tier zuckt. Es werden jetzt die Füße von den Fesseln befreit. Das Tier bleibt ungefesselt ruhig liegen bis zum Schluß des Versuches. Nur der Kopf bleibt im Halter befestigt.

$6 \mathrm{~h} .39 \mathrm{~m}$.

$$
\begin{array}{llllllll}
250 & 200 & 200 & 200 & 250 & 250 & 200 & 200 \\
350 & 250 & 250 & 200 & 300 & 250 & 250 &
\end{array}
$$

Frequenz: 65 Atemzüge pro Minute.

Volumen der ausgeatmeten Luft:

$$
\text { in } 1 \text { Minute } 480,00 \text { in } 1 \text { Atemzug } 7,38 \text {. }
$$

$6 \mathrm{~h} .48 \mathrm{~m}$.

$$
\begin{array}{lllll}
250 & 250 & 375 & 225 & 250 \\
250 & 225 & 250 & 175 & 250 .
\end{array}
$$

Frequenz: 66 Atemzinge pro Minute.

Volumen der ausgeatmeten Luft:

in 1 Hinute 500,00 in 1 Atemzug 7,58.

$6 \mathrm{~h} .55 \mathrm{~m}$. Weitere Injektion von $1 \mathrm{ccm}$ der 10 proz. Epiosinchloridlösung (=0,1 Epiosinchlorid). Daner der Injektion 1/2 Minute.

$6 \mathrm{~h} .58 \mathrm{~m}$.

$$
\begin{array}{lllll}
200 & 225 & 200 & 150 & 200 \\
200 & 225 & 250 & 200 & 200
\end{array}
$$

Frequenz: 51 Atemzuge pro Minute.

Volumen der ausgeatmeten Luft:

in 1 Minute 410,00 in 1 Atemzug 8,04.

$7 \mathrm{~h} .16 \mathrm{~m}$. Das Tier wird mit Chloroform getötet.

$$
\text { Versuch Nr. V. }
$$

Graues, männliches Kaninchen. $2400 \mathrm{~g}$. Sebr wild und sehr empfindlich. Tracheotomiert und in die rechte Jugularvene eine Kantile eingebunden. 
Die chemische Konstiution des Morphins in ihrer Beziehung zur Wirkung. 129

$5 \mathrm{~h} .30 \mathrm{~m}$. Operation beendigt.

$\begin{array}{llllll}5 \text { h. } & 45 \mathrm{~m} \text {. } \quad 240 & 200 & 190 & 175 & 180\end{array}$

$\begin{array}{lllll}140 & 150 & 185 & 200 & 180\end{array}$

Frequenz: 68 Atemzüge pro Minute.

Volumen der ausgeatmeten Luft:

in 1 Minute 384,00 in 1 Atemzug 5,65.

$6 \mathrm{~h} .2 \mathrm{~m}$. Injektion von 1 cem einer 10 proz. Lösung von Epiosinchlorid (=0,1 Epiosinchlorid). Dauer der Injektion 3/4 Minute.

$6 \mathrm{~h} .5 \mathrm{~m}$.

$\begin{array}{rrrrrrrr}180 & 150 & 210 & 160 & 180 & 170 & 150 & 180 \\ 200 & 180 & 180 & 180 & 190 & 200 & 170 & \end{array}$

Frequenz: 65 Atemzüge pro Minute.

Volumen der ausgeatmeten Luft:

in 1 Minute 357,32 in 1 Atemzug 5,50 .

$6 \mathrm{~h} .17 \mathrm{~m}$. Es werden jetzt die Füße von den Fesseln befreit. Das Tier bleibt ungefesselt bis zum Schluß des Versuches liegen. Die Augen sind geschlossen. Sämtliche Pfoten und der Schwanz können mit einem Nadelhalter zusammengepreßt werden, so daß die Knochen knacken, ohne daß das Tier nur im geringsten zuckt.

$6 \mathrm{~h} .21 \mathrm{~m}$.

$\begin{array}{llllllllll}120 & 240 & 150 & 210 & 210 & 220 & 220 & 200 & 230 & 230 \\ 210 & 230 & 220 & 200 & 210 & 210 & 200 & 200 & 220 & 220\end{array}$

Frequenz: 70 Atemzüge pro Minute.

Volumen der ausgeatmeten Luft:

$6 \mathrm{~h} .36 \mathrm{~m}$.

$$
\text { in } 1 \text { Minute } 415,00 \text { in } 1 \text { Atemzug } 5,93 \text {. }
$$

$$
\begin{array}{llllllll}
200 & 260 & 240 & 220 & 240 & 250 & 230 & 230 \\
200 & 220 & 230 & 250 & 260 & 290 & 280 &
\end{array}
$$

Frequenz: 80 Atemzüge pro Minute,

Volumen der ausgeatmeten Luft:

in 1 Minute 480,00 in 1 Atemzug 6,00.

$6 \mathrm{~h} .48 \mathrm{~m}$. Weitere Injektion von $1 \mathrm{ccm}$ der 10 proz. Epiosinchloridlösung (=0,1 Epiosinchlorid). Dauer der Injektion $1 / 2$ Minute.

$6 \mathrm{~h} .50 \mathrm{~m}$.

$\begin{array}{llllllll}120 & 140 & 130 & 130 & 140 & 160 & 140 & 170 \\ 130 & 120 & 100 & 120 & 140 & 100 & 140 & \end{array}$

Frequenz: 64 Atemzüge pro Minute.

Volumen der anggeatmeten Luft:

$7 \mathrm{~h} .05 \mathrm{~m}$.

$$
\text { in } 1 \text { Minute 264,00 in } 1 \text { Atemzug } 4,13 \text {. }
$$

$$
\begin{array}{rrrrrrrr}
110 & 90 & 110 & 80 & 90 & 100 & 110 & 90 \\
90 & 100 & 100 & 120 & 110 & 100 & 110 & .
\end{array}
$$

Frequenz: 53 Atemzüge pro Minute.

Volumen der ansgeatmeten Luft:

in 1 Minute 201,32 in 1 Atemzug 3,81. 
$\begin{array}{llllll}7 \text { h. } 24 \mathrm{~m} . & 100 & 100 & 110 & 120 & 150\end{array}$

$\begin{array}{lllll}90 & 90 & 120 & 120 & 120\end{array}$

Frequenz: 56 Atemzüge pro Minute.

Volumen der ansgeatmeten Luft:

in 1 Minute 200,00 in 1 Atemzig 3,57.

$7 \mathrm{~h} .45 \mathrm{~m}$. Narkose vollkommen. Pupillen nicht verengt. Die Schwanzwurzelknochen können mit einem Nadelhalter zermalmt werden, ohne daß eine Reaktion von seiten des Tieres erfolgt. Es wird mit Chloroform getötet.

\section{Versueh Nr. VI.}

Weißes, gelbgeflecktes Kaninchen, weiblich, $2550 \mathrm{~g}$ schwer. Tracheotomiert, Kanutle in eine Jugularvene eingebunden.

$4 \mathrm{~h} .46 \mathrm{~m}$. Operation beendigt.

5 h. $02 \mathrm{~m}$.

$\begin{array}{lllllllll}350 & 400 & 450 & 300 & 370 & 350 & 350 & 350 & 400 \\ 300 & 300 & 400 & 350 & 330 & 350 & 350 & 350 & 400 \\ 520 & 370 & 350 & 350 & & & & & \\ 430 & 330 & 350 & & & & & & \end{array}$

Frequenz: 46 Atemzinge pro Minute.

Volumen der ausgeatmeten Luft:

in 1 Minute 732,00 in 1 Atemzug 15,91 .

$5 \mathrm{~h} .17 \mathrm{~m}$. Injektion von $1 \mathrm{ccm}$ einer 10 proz. Epiosinchloridlösung $(=0,1$ Epiosinchlorid). Dauer der Injektion $1 / 2$ Minute.

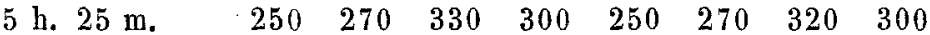

$\begin{array}{lllllll}300 & 350 & 250 & 230 & 300 & 230 & 300\end{array}$

Frequenz: 48 Atemzüge pro Minute.

Volumen der ausgeatmeten Luft:

in 1 Minute 566,66 in 1 Atemzug 11,81 .

Die fast vollkommen geschlossenen Augen sind mit Tränen gefüllt. Die Füße werden von den Fesseln befreit, nur der Kopf bleibt im Halter befestigt. So bleibt das Tier ruhig liegen bis zum Sehluß des Versuches, Hinter- und Vorderpfoten können mit einem Nadelhalter so heftig zu= sammengepreßt werden, daß die Knochen knacken. Es erfolgt keine Reaktion von seiten des Tieres.

$5 \mathrm{~h} .40 \mathrm{~m}$.

$\begin{array}{llllllllll}300 & 300 & 320 & 350 & 270 & 280 & 270 & 340 & 250 & 300 \\ 250 & 380 & 330 & 350 & 300 & 350 & 290 & 280 & 300 & 230\end{array}$

Frequenz: 48 Atemzinge pro Minute.

Volumen der ausgeatmeten Luft:

in 1 Minute 604,00 in 1 Atemzug 12,58.

$6 \mathrm{~h} .-\mathrm{m}$.

$\begin{array}{llllllllll}450 & 380 & 330 & 330 & 300 & 300 & 400 & 380 & 370 & 240\end{array}$

$\begin{array}{llllllllll}320 & 270 & 320 & 350 & 350 & 300 & 270 & 350 & 310 & 380\end{array}$

Frequenz: 36 Atemzïge pro Minute.

Volumen der ausgeatmeten Luft:

iu 1 Minute 670,00 in 1 Atemzug 18,61 . 
Die Betrachtung der mitgeteilten Versuche lehrt deutlich, daß das Epiosin, indem es sowohl das Atemvolum wie die Atemfrequenz, und zwar in einer solchen Relation dieser beiden Größen vermindert, daß gleichzeitig auch die durch den einzelnen Atemzug geförderte Luftmenge abnimmt, die Respirationstätigkeit erheblich herabzusetzen imstande ist. Dieses Verhalten kommt außerordentlich klar in dem Versuch Nr. IV zur Erscheinung, ist aber auch in den beiden anderen Versuchen, die freilich noch einige Besonderheiten darbieten, auf die ich gleich zu sprechen kommen werde, nicht zu verkennen. Ganz ebenso wirkt das Morplin auf die Atmung. Speziell für Kaninchen ist dies von Leichtenstern ${ }^{1}$ ), der ebenso wie ich die Volumina der einzelnen Atemztige in seiner Arbeit verzeichnet hat, sowie Wilmanns ${ }^{2}$ ) und Stursberg ${ }^{3}$ ) ermittelt worden, die sich auf die Messung der Atemvolumina beschränkt haben.

In Versuch Nr. VI sieht man die Atemfrequenz statt verringert um ein Geringes vermehrt, aber gleichzeitig sowohl das in der Zeiteinheit wie das mit dem einzelnen Atemzuge ausgeatmete Luftquantum vermindert. Zum Sehluß tritt aber ein gerade umgekehrtes Verhalten ein. Die Atemfrequenz ist erheblich herabgesetzt, dagegen das Volumen der pro Minute und in noch sehr viel höherem Maße die Menge der pro Atemzug ausgeatmeten Luft vermehrt. In dieser Vergrößerung des Atemvolums durch Epiosin kann man eine Analogie mit der Kodeinwirkung sehen. Denn Stursberg ${ }^{4}$ ) hat gezeigt, dab nach Injektion von Kodein in gewissen Dosen bei Kaninchen die Atemvolumina nur vorübergehend abfielen, nach kurzer Frist dagegen wieder zur Norm oder in einem Versuch ${ }^{5}$ ) sogar darüber anstiegen. Ich habe schon in meiner ersten Arbeit ${ }^{6}$ ) wegen eigenartiger Krämpfe, die es im Anschlub und im Verlauf der narkotischen Wirkung bei Hunden veranlassen kann, das Epiosin mit dem Kodein verglichen. Bei Kaninchen habe ich aber weder in den mitgeteilten, noch in einigen anderen nicht protokollierten Versuchen nach Epiosininjektion Krämpfe auch nur andeutungsweise auftreten sehen. Vielleicht beschränkt sich demnach die zentral-

1) Leichtenstern, I. c. 197.

2) C. Wilmanns, Die direkte Erregung des Atmungszentrums durch den. Weingeist. Pflügers Archiv. LXVI. (1897.) 189.

3) H. Stursberg, Über die Wirkung einiger Abkömmlinge des Morphins. anf die Atmung. Archives internationales de Pharmacodynamie. IV. (1898.) 333

4) Dersel be, ebenda. 334 .

5) Ibid. S. 335 .

6) Vahlen, Chemische Konstitution des Morphins usw. Dieses Archir. XLVII. (1902.) 408. 
erregende Wirkung, die das Epiosin ebenso wie das Morphin und in noch höherem Grade das Kodein neben der narkotischen besitzt, bei Kaninchen auf die beschriebene Veränderung der Atmung.

In Versuch Nr. V wurde gerade umgekehrt wie in Versuch Nr. VI nach der Epiosininjektion vor der Herabsetzung der Respirationsenergie eine freilich recht geringe Steigerung beobachtet. Ob es sich dabei wirklich um eine primäre Erregung des Atemzentrums oder um eine zufällige, nicht weiter aufgeklärte Komplikation des Experimentes handelte, muß dahingestellt bleiben.

Bezüglich des Einflusses, den das Epiosin auf die Respiration des Hundes ausübt, konnte ich bereits in meiner ersten Mitteilung ${ }^{1}$ ) auf Grund von Atmungskurven, die am tracheotomierten Tier mittelst cines Mareyschen Tambours aufgezeichnet worden waren, das Urteil aussprechen, daß die Atmung durch den Einfluß des Epiosins langsamer, flacher und regelmäßiger wird̄. Bei Bunden weist die Atmung normalerweise häufig eine große Unregelmäßigkeit des Rbythmus auf. In ganz besonders hohem Grade ist dies unmittelbar nach dem Luftröhrenschnitt und der Einführung einer Kanüle in die Trachea der Fall. Es wechseln dann Perioden miteinander ab, die bald von angestrengten und beschleunigten, bald von langsamen und ruhigen Atemzügen gebildet werden. Oft verstreicht eine geraume Zeit, ehe eine gewisse Regelmäßigkeit des Rhythmus eingetreten ist, die einen einigermaßen brauchbaren Mittelwert für das Atemvolum zu gewinnen erlanbt. Mit einem Schlage sieht man aber nach intravenöser Injektion von 0,2 Epiosinchlorid eine vorher noch so unregelmäßige Respiration ruhig und gleichmäßig werden. Im einzelnen ist die Veränderung der Atmung, wie oben gesagt und wie sie sich bei Kaninchen nach den geschilderten Experimenten darstellte, d. h. Atemfrequenz und Atemvolum nehmen ab und zwar unter einer derartigen Verschiebung ihrer Proportion, dab auch der einzelne Atemzug an Ergiebigkeit einbübt. Im folgenden ist ein Hundeversuch ausführlich mitgeteilt. Die Anordnung ist genau dieselbe wie bei den Kaninchenversuchen.

\section{Versuch Nr. VII.}

Hund, $6850 \mathrm{~g}$ schwer. Tracheotomiert und eine Kanüle in eine Jugularvene eingeführt.

$4 \mathrm{~h} .30 \mathrm{~m}$. Operation beendigt.

5 h. $-\mathrm{m}$.

$\begin{array}{rrrrrrr}2600 & 1100 & 1300 & 1500 & 1000 & 1750 & 1900 \\ 1800 & -1000 & 1000 & 1500 & 1700 & 1650 & 1450\end{array}$

1) L. c. S. $40 b$. 
Die chemische Konstitution des Morphins in ihrer Beziehung zur Wirkung. 133

Frequenz: 40 Atemzlige pro Minute.

Volumen der ausgeatmeten Luft:

in 1 Minute 3133,32 in 1 Atemzug 78,33 .

$5 \mathrm{~h} .20 \mathrm{~m}$. Injektion von $2 \mathrm{ccm}$ einer 10 proz. Lösung von Epiosinchlorid (=0,2 Epiosinchlorid). Dauer der Injektion 2 Minuten. Bald darauf Kotentleerung. Wird an sämtlichen Füßen seiner Fesseln entledigt. Bleibt ungefesselt, nur den Kopf im Halter befestigt, rubig bis zum Schluß des Versuches liegen.

$\begin{array}{rrrrr}5 \mathrm{h.} 24 \mathrm{m.} & 950 & 1050 & 950 & 950 \\ 1000 & 900 & 1100 & 1100 \\ 950 & 1050 & 950 & 900 \\ 900 & 950 & 900 & 950 \\ 1000 & 900 & 900 & 900\end{array}$

Frequenz: 30 Atemzüge pro Minute.

Volumen der ausgeatmeten Luît:

in 1 Minute 1925,00 in 1 Atemzug 64,00.

Man kann Nadeln durch Nase und Ohren stechen, ohne daß eine Reaktion von seiten des Tieres erfolgt. Es geschieht dies ebensowenig; wenn die Ohren mit einem Nadelhalter bis zum vollkommenen Schluß der Branchen geklemmt werden. Pupillen nicht verändert, jedenfalls nicht verengt.

5 h. $39 \mathrm{~m}$

$\begin{array}{lllllll}950 & 750 & 850 & 850 & 850 & 900 & 800 \\ 850 & 900 & 900 & 850 & 900 & 850 & 900 \\ 650 & 850 & 750 & 800 & 900 & 900 & 950 \\ 750 & 700 & 850 & 750 & 900 & 800 & 950 \\ 850 & 850 & 750 & 850 & 850 & 900 & 800\end{array}$

Frequenz: 28 Atemzüge pro Minute.

Volumen der ausgeatmeten Luft:

in 1 Minute 1682,86 in 1 Atemzug 60,10 .

5 h. $57 \mathrm{~m}$. Man kann Nadeln durch Ohren und Nase stecken, olne daß eine Reaktion eintritt. Ebenso kann man die Ohren wiederholt mit einem Nadelhalter bis zum vollkommenen Schluß der Branchen klemmen, ohne.daß das Tier, das immer noch ungefesselt daliegt, zuckt.

$6 \mathrm{~h} .05 \mathrm{~m}$.

$\begin{array}{rrrr}950 & 1000 & 950 & 1000 \\ 950 & 950 & 900 & 1000 \\ 1000 & 1000 & 850 & 950 \\ 1000 & 1000 & 1000 & 1000 \\ 950 & 1000 & 1000 & 950\end{array}$

Frequenz: 26 Atemzïge pro Minute.

Volumen der ausgeatmeten Luft:

in 1 Minute 1940,00 in 1 Atemzug 69,29.

Wird mit Chloroform getötet. Es sei hier hinzugefügt, daß dieser Hund weder unmittelbar nach der Injektion des Epiosins die geringste Erregung zeigte noch im weiteren Verlauf.

Auch in diesem Hundeversuch (ebenso wie in dem Kaninchenversuch Nr. VI) sieht man zum Schluß das Atemvolum wieder etwas 
ansteigen, wenn es auch noch weit unter dem Normalwert bleibt. Im ganzen findet sich die geschilderte Wirkung des Epiosins auf die Respiration des Hundes in guter Übereinstimmung mit derjenigen des Morphins, wie man sie in den Experimenten von Brinda ${ }^{1}$ ) dargestellt findet. Freilich bat es auch dieser Autor unterlassen, die Volumina der einzelnen Atemzäge mitzuteilen. Da er aber in einigen seiner Versuchsprotokolle außer den Atemvolumina (für die Zeiteinheit) auch die Atemfrequenz verzeichnet hat, so ist man imstande, die Volumina der Atemzüge zu berechnen. Es ergibt sich dann aus Brindas Experimenten $12 \mathrm{a}$ und $6 \mathrm{a}^{2}$ ), daß Hunde unter der Einwirkung des Morphins nicht nur weniger Luft in der Zeiteinheit ausatmeten, sondern daß bei ihnen infolge der noch raseher als die Atemrolumina gesunkenen Atemfrequenz auch die dureh die einzelnen Atemzüge geförderten Luftmengen abnahmen. Dagegen zeigte der Hund in Versuch $8 \mathrm{a}^{3}$ ) nach einer größeren Morphindosis eine Steigerung der Atemfrequenz. Ob diese aber von einer Erhöhung der Zeit- und der Atemvolumina begleitet war, das kann man, da nichts darüber angegeben wird, nicht wissen. Immerhin ist es aber möglich, wenn nicht sogar wahrscheinlich. Es würde dann die der anfänglichen Depression folgende, übrigens geringe und nicht über den Normalwert hinaufgehende Steigerung der Respirationsenergie in meinem Versuch Nr. VII einer Analogie mit der Morphinwirkung keinesfalls widersprechen.

\section{Die Wirkung des Epiosins auf den Blutkreislauf.}

Bezüglich der Veränderung, die der Blutdruck unter dem Einflub des Epiosins erleidet, habe ich bereits in meiner ersten Arbeit ${ }^{4}$ ) drei Versuche an Hunden mitgeteilt. In zwei Fällen stieg der Blutdruck deutlich an, im dritten blieb er unverändert. Ich hielt es nun nicht für ausgeschlossen, daß die beobachtete Druckerhöhung lediglich die Folge eines gleichzeitig auftretenden leisen Tremors gewesen sei. Man sieht zwar nicht selten, ohne daß man einen bestimmten Grund anzugeben wüßte, namentlich junge Hunde, sobald sie auf den Operationstisch gespannt sind, gleichsam von einem Frostschauer ergriffen werden, auch wenn sie vor Abkühlung sorg-

1) Antonio Brinda, Sull 'azione respiratoria della morfina e di alcuni suoi succedanei. Archives internationales de Pharmacodynamie et de Thérapie. IX. (1901.) 64.

2) Ibid. S. 67.

3) Ibid. S. 68 .

1) L. c. S. 403,404 u. 407. 
Die chemische Konstitution des Morphins in ihrer Beziehung zur Wirkung. 135

fältig geschützt sind. In meinen Experimenten war aber dieses Zittern wohl stets ein Vorbote der heftigeren klonischen Zuckungen, die namentlich nach großen Epiosindosen im weiteren Verlauf der Vergiftung nie auszubleiben pflegen. Um daher eine eventuelle Blutdruckerhöhung sicher als eine primäre Wirkung des Epiosins zu erkennen, wurden weitere Versuche an kuraresierten Tieren angestellt.

\section{Versuch Nr. VIII.}

Hund, $7600 \mathrm{~g}$ schwer.

\begin{tabular}{|c|c|c|c|}
\hline Zeit & $\begin{array}{l}\text { Blutdruck } \\
\text { in mm } \\
\text { Queck silber }\end{array}$ & Pulsfrequenz & Bemerkungen \\
\hline $12 \mathrm{~h} .05 \mathrm{~m}$. & - & - & $\begin{array}{l}\text { Tracheotomie, kunstliche Atmung. In- } \\
\text { jektion einer Kurarelösung in eine Jugu- } \\
\text { larvene. }\end{array}$ \\
\hline $\begin{array}{l}12 \mathrm{~h} .35 \mathrm{~m} .- \\
12 \mathrm{~h} .40 \mathrm{~m} .\end{array}$ & 85 & 146 & 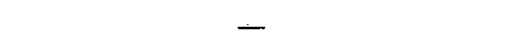 \\
\hline $12 \mathrm{~b} .50 \mathrm{~m}$. & - & - & $\begin{array}{l}\text { Intravenöse Injektion von } 3 \mathrm{ccm} \text { einer } \\
10 \text { proz. Epiosinchloridlösung }(=0,3 \text { Epio- } \\
\text { sinchlorid. Die Dauer der Injektion } 1 \text { Min. } \\
\text { Während der Injektion sinkt der Blut- } \\
\text { druck fast bis auf die Abszissenachse, steigt } \\
\text { aber alsbald wieder. }\end{array}$ \\
\hline $\begin{array}{l}12 \text { h. } 52 \mathrm{~m} \text {. } \\
1 \text { h. } 15 \mathrm{~m} \text {. } \\
1 \text { b. } 50 \mathrm{~m} .\end{array}$ & $\begin{array}{l}142 \\
109 \\
101\end{array}$ & $\begin{array}{l}126 \\
127 \\
139\end{array}$ & - \\
\hline
\end{tabular}

Versuch Nr. IX.

Pudel, 9 kg. schwer.

\begin{tabular}{|c|c|c|c|}
\hline Zeit & $\begin{array}{l}\text { Blutdruck } \\
\text { in mm } \\
\text { Quecksilber }\end{array}$ & Pulsfrequenz & Bemerkungen \\
\hline $11 \mathrm{~h} .50 \mathrm{~m}$. & 88 & 118 & $\begin{array}{l}\text { Tracheotomiert, kúnstliche Atmung, } \\
\text { kuraresiert. Mittlerer Druek vor der } \\
\text { Epiosininjektion. } \\
\text { Intravenöse Injektion von } 4 \text { ccm einer } \\
\text { 10proz. Epiosinchloridlösung ( }=0,4 \text { Epiosin- } \\
\text { chlorid). Dauer der Injektion } 1 \text { Min. Wäh- } \\
\text { rend der Injektion gehtder Druck fast bis zur } \\
\text { Abszisse herab. }\end{array}$ \\
\hline $\begin{array}{l}11 \mathrm{~h} .53 \mathrm{~m} . \\
12 \mathrm{~h} .06 \mathrm{~m} . \\
12 \mathrm{~h} .47 \mathrm{~m} . \\
1 \text { h. } 20 \mathrm{~m} .\end{array}$ & $\begin{array}{r}73 \\
101 \\
91 \\
83\end{array}$ & $\begin{array}{l}108 \\
126 \\
154 \\
183\end{array}$ & $\begin{array}{r}- \\
- \\
-\end{array}$ \\
\hline
\end{tabular}


Versuch Nr. X.

$\mathrm{Hund}, 6 \mathrm{~kg}$ schwer.

\begin{tabular}{|c|c|c|c|}
\hline Zeit & $\begin{array}{l}\text { BIutdruck } \\
\text { in mm } \\
\text { Quecksilber }\end{array}$ & Pulsfrequenz & Bemerkungen \\
\hline & & & $\begin{array}{l}\text { Tracheotomie, ktunstliche Atmung, intra- } \\
\text { venöse Injektion von Kurarelösung. }\end{array}$ \\
\hline $\begin{array}{l}11 \text { h. } 20 \mathrm{~m} .- \\
11 \text { h. } 35 \mathrm{~m} .\end{array}$ & 80 & 120 & - \\
\hline $11 \mathrm{~h} .45 \mathrm{~m}$. & - & - & $\begin{array}{l}\text { Intravenöse Injektion von } 1 \mathrm{ccm} \text { einer } \\
\text { 10proz, Epiosinchloridlösung }(=0,1 \text { Epio- } \\
\text { sinchlorid). Dauer der Injektion } 1 \text { Min. } \\
\text { Wuhrend der Injektion geht der Blutdruck } \\
\text { bis zur Abszisse herab, erhebt sioh aber } \\
\text { alsbald wieder. }\end{array}$ \\
\hline $11 \mathrm{~h} .47 \mathrm{~m}$. & 137 & 120 & \\
\hline $11 \mathrm{~h}$. & - & - & $\begin{array}{l}\text { Abermalige Injektion von } 1 \mathrm{ecm} \text { der } \\
10 \text { proz. Epiosinehloridlösung. Wiederum } \\
\text { geht der Druck fast bis zur Abszisse herab, } \\
\text { steigt aber alsbald wieder. }\end{array}$ \\
\hline 1 h. $.51 \mathrm{~m} .10 \mathrm{~s}$ & 80 & 120 & - \\
\hline & 109 & 10 & - \\
\hline
\end{tabular}

Versuch Nr. XI.

Foxterrier von $7,5 \mathrm{~kg}$ Gewicht.

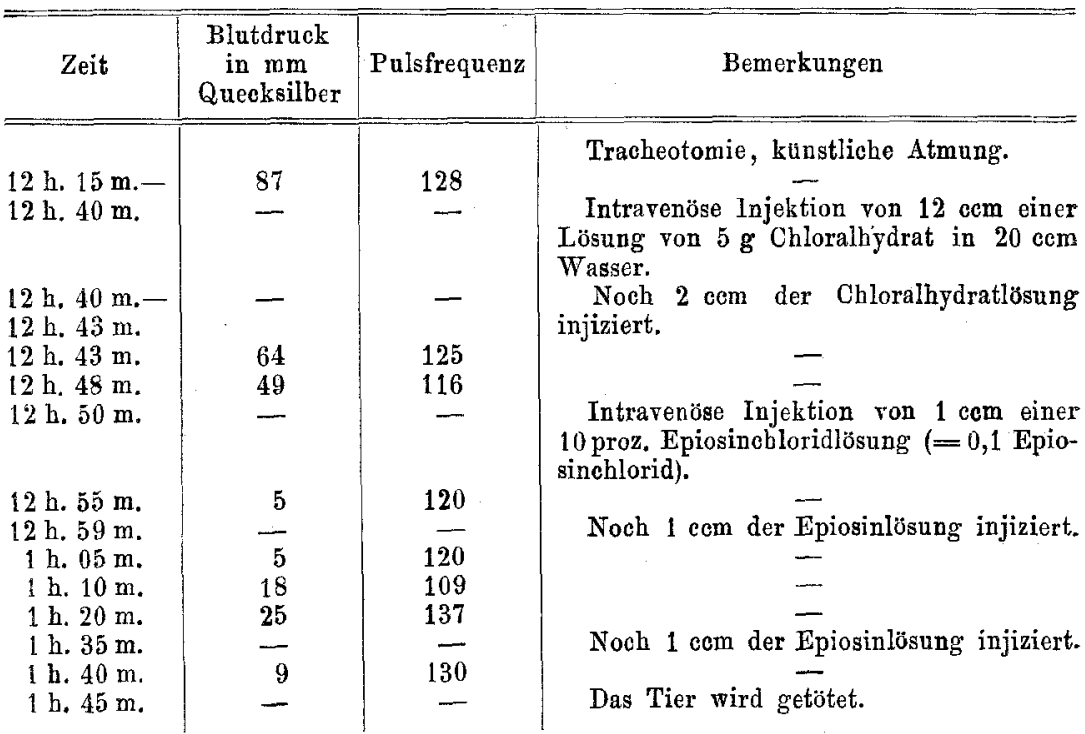

Die Hauptwirkung des Epiosins auf den Blutdruck beruht, wie aus den Versuchen Nr. VIII, IX und X leicht zu erkennen, in einer zum Teil sehr beträchtlichen Erhöhung. Der Steigerung des Blut- 
drucks geht jedoch stets eine erbebliche Depression voraus, die aber nur von ganz kurzer Dauer ist. So sieht man in Versuch Nr. VIII bereits eine Minute nach beendigter Epiosininjektion den Blutdruck wieder um 66 Proz. des ursprünglichen Wertes erhöht. In Versuch Nr. IX danert diese Depression zwar etwas länger an, immerhin ist eine Viertelstunde nach der Epiosininjektion der Druck im Gefäßsystem um ca. 15 Proz. seiner anfänglichen Größe vermehrt. In Versuch $\mathrm{X}$ ist die Zeitdauer der plötzlichen Druckverminderung nach der zweiten Epiosininjektion genan verzeichnet. Es ergibt sich daraus, daß schon 1 Minute 10 Sekunden nach Beginn der Injektion der Blutdruck die normale Höhe wieder erreicht hatte und eine Viertelstunde später beträchtlich darüber binausgestiegen war. Es fragte sich, ob die durch Epiosin bewirkte Blutdruckerhöhung nicht vielleicht auf einer Steigerung der Propulsivkraft des Herzens beruhte. Deshalb wurde in Versuch VI vor der Applikation des Epiosins der intraarterielle Druck durch Chloralhydrat erheblich berabgesetzt. Da nun die darauffolgenden Epiosininjektionen den Blutdruck nicht mehr in die Höhe zu treiben vermochten, so darf man schließen, daß das Epiosin den Blutdruck wabrscheinlich durch Vermittelung des vasomotorischen Zentrums erhöht, aber nicht die Leistungsfähigkeit des Herzens zu steigern vermag.

Die geschilderte Wirkung des Epiosins auf den Blutdruck scheint keine Analogie in dem Verhalten des Morphins zu finden. Denn dieses Alkaloid äußert in den durchschnittlich in Betracht kommenden Dosen keinen nennenswerten Einfluß auf das Kreislaufsystem, wo es aber einen solchen zur Geltung bringt, nach außerordentlich großen Dosen oder gegen Ende einer letalen Vergiftung, setzt es den Blutdruck durch Lähmung des Herzens und der Gefäße herab. Speziell für mein Versuchstier, den Hund, beweisen dies einige Experimente ron Gscheidlen ${ }^{1}$, , besonders deutlich Versuch Nr. XVI ${ }^{2}$, in dem ein Hund nach intravenöser Applikation von 0,2 Morphin. acetic. in $13 / 4$ Stunden verendete. Aber es ist interessant, festzustellen, daß derselbe Autor bei Kaninchen ${ }^{3}$ ) nach intravenöser Applikation von Morphin. acetic. auch Erhöhung des Blutdruckes beobachten konnte.

1) Gscheidlen, Untersuchungen aus dem physiologischen Institut in Würzburg. 1I. 1. Leipzig 1869.

2) L. c. S. 31 .

3) Vgl. l. c. Vers. X S. 136, XII. S. 27 und XIII S. 30. 
IIr. Über die narkotische Wirkung des Epiosins.

In meiner ersten Arbeit wurde in einer Reihe von Versuchen gezeigt, daß Hunde nach intravenöser Applikation von einem bis mehreren Dezigrammen Epiosinchlorid in einen narkotisehen Zustand geraten, der als morphiumähnlich bezeichnet werden mußte. In dieser Hinsicht waren die mitgeteilten Beobachtungen von so un. zweideutiger Beweiskraft, daB, welche Modifikationen in einzelnen Zügen das von mir entworfene Bild der Epiosinwirkung durch weitere Tierversuche anch erfabren mag, seine Richtigkeit in der Hauptsache nicht erschittert werden kann. In der Tat ist auch bis jetzt, beinahe anderthalb Jahre nach dem Erseheinen meiner Arbeit, ron pharmakologischer Seite gegen meine Art zu experimentieren und zu argumentieren nicht der geringste Einwand erhoben worden.

Um so befremdlicher war es mir, als ich bereits drei Monate nach dem Erscheinen meiner ersten Publikation in einer rein chemischen Untersuchung von R. Pschorr ${ }^{1}$ ) die bündige Erklärung las: "Das Epiosin ist ein starkes Blutgift, indem sich bei Warmbltitern die Bildung von Methämoglobin nachweisen lief. Die "morphin"ähnliche Wirkung ist daher a f die Blutveränderung und nicht, wie bei Morphin, auf die Nervenwirkung zuriuckzuführen, soda $B$ ein Vergleich völlig a ugeschlossen ist." Dieses Verdikt über meine Versuche wurde ausgesprocben ohne jede experimentelle Begrïndung.

Und doch war Pschorrs Urteil, wenn richtig, spielend leicht zu beweisen. Es waren nur folgende Feststellungen zu machen: 1. Bewirkt das Epiosin im Blute lebender Hunde Methämoglobinbildung? 2. Trifft diese behanptete Methämoglobinbildung im Organismus des lebenden Hundes stets mit der narkotischen Wirkung zusammen? 3. Hat diese konstant gleichzeitig mit der narkotischen Wirkung zu beobachtende Methämoglobinbildung wirklich einen so hohen Grad, um als Erklärung für eine narkotische Wirkung beim Hunde angesehen werden zu können. Denn davon könnte ja auch im entferntesten nicht die Rede sein, daß eine zwar regelmäßig auftretende, aber nicht sehr beträchtliche, etwa gar nur mittelst des Spektroskops eben nachweisbare Methämoglobinbildung einen narkotischen Zustand veran-

1) R. Pschorr, Über das 9-Amino-10-Oxypheranthren (Vahlens „Morphigenin") und 9.10-Diaminophenanthren." Bericht d, deutschen chem. Gesellschaft. XXXV. (1902.) 2729. 
lassen sollte. Selbst bei einer so hochgradigen Blutveränderung durch Methämoglobinbildung, daß sie sich durch Bräunung des Blutes, livide Färbung der Schleimhäute usw. dem unbewaffneten Auge, ja sogar dem ungeübten Blick jedes Laien auf das Unzweideutigste dokumentiert, darf man darüber im Zweifel sein, ob ein vorhandener narkotischer Zustand $\mathrm{nur}$ eine Folge jener Blutveränderung oder vielmehr eine davon unabhängige, selbständige Wirkung ist, seitdem Binz ${ }^{1}$ ) durch sorgfältige Analyse der Erscheinungen gezeigt hat, daß die durch das salpetrigsaure Natron, dessen methämoglobinbildende Kraft auch innerhalb des Organismus eine enorme ist, hervorgebrachte narkotische Wirkung ganz und gar nichts mit der Zerstörung des Blutfarbstoffes zu tun hat.

Es wird nach dem Gesagten einleuchten, daß in dem vorliegenden Streit, ob die von mir an Hunden beobachtete narkotische Wirkung des Epiosins einer angeblichen Zersetzung des Blutfarbstoffes zugeschrieben werden muß oder nicht, die Entscheidung von der Beantwortung jener drei Fragen (oder der dritten allein, die ja die beiden andern in sich schließt) abhängt. Und zwar sind diesedrei Fragenklippundklarmitja oder nein zubeantworten. Eindrittes gibtes nicht.

Ich habenun obigedrei Fragen in zahlreichen Experimenten mit nein beantworten müssen. Aus meiner ersten Arbeit ist zu ersehen ${ }^{2}$, daß ich allen Hunden bis auf einen das Epiosin intravenös appliziert habe. Da ich nicht wissen konnte, wie groh die zu injizierende Menge im einzelnen Falle sein mußte, um die volle Wirkung zu erzielen, blieb natürlich die Kanüle geraume Zeit im Blutgefäße eingebunden liegen. Ich hatte also Wundfläche und Blut dauernd vor Augen, ich branchte nach einer Veränderung des Blutfarbstoffes, wenn sie eintrat, nicht noch besonders zu suchen. Ferner hatte ich in der Mehrzahl der Experimente mit blutigen Operationen anderer Art zu tun, die mir in jedem Falle im Verlaufe von Stunden, ohne daß ich besonders darauf fabndete, die Beschaffenheit des Blutes meinen Blicken darboten. Aber niemals zeigte das Blut eine Beschaffenheit, die auch nur den geringsten Verdacht auf eine Anwesenheit von Methämoglobin hätte erwecken können, geschweige denn in solcher Menge, wie sie allein

1) Binz, Über einige neue Wirkungen des Natriumnitrits. Dieses Archiv. XIII. (1881.) 133. - Derselbe, Narkotische Wirkungen von Hydroxylamin und Natriumnitrit. Virchows Archiv. 118. (1889.) 121. - Derselbe, Toxikologisches über das Hydroxylamin. Virchows Archiv. 113. (1888.) 1.

2) L. c. S. $402-408$. 
dafür in Betracht kommen konnte, wenn man sie als Ursache der beobachteten narkotischen Wirkung ansehen wollte.

Als ich daher die bereits zitierte, ohne Anführung eines einzigen Tierexperiments ausgesprochene Erklärung Pschorrs las, war ich keinen Augenblick in Zweifel darüber, daß es sich nur um einen Irrtum handeln konnte. Die nächstliegende Vermutung: war, daß Pschorr von einem Reagensglasversuch auf das Verhalten der Substanz im lebenden Organismus geschlossen habe. In der Tat bildet sich beim Vermischen einer konzentrierten Blutlösung mit einer konzentrierten Lösung von Epiosinchlorid alsbald ein klumpiger Niedersehlag und die darüberstehende Flüssigkeit färbt sich unter Methämoglobinbildung nach einiger Zeit braun. Arbeitet man aber mit größeren Verdünnungen, so können einige Stunden vergehen, ehe Methämoglobin nachweisbar ist. Sehr grob ist also die methämoglobinbildende Kraft des Epiosins (verglichen z. B. mit der des Ferricyankaliums oder des Natriumnitrits) auch im Reagensglase nicht. Genauere Untersuchungen habe ich aber diesem Gegenstande nicht widmen wollen; denn es ist für die Beurteilung meiner Epiosinversuche an Tieren vollkommen gleichgültig, ob und in welchem Grade das Epiosin eine Veränderung des Blutfarbstoffes im Reagensglase bewirkt. Substanzen, die zwar im Glase Methämoglobinbildung veranlassen, im lebenden Organismus sich aber vollkommen indifferent gegen 'den Blutfarbstoff verhalten, gibt es in großer Zahl. Ich habe darauf bereits in einer kurzen Erwiderung auf Psohorrs Angriff ausdrücklich hingewiesen 1).

Da meine Versuchstiere, wie bereits angegeben, eine mit bloßem Auge wahrnehmbare Veränderung ihrer Blutfarbe nicht darboten, die auch nur im geringsten dem Verdacht hätte Raum geben können es wäre Methämoglobin in ihm enthalten, so lag keine Veranlassung vor, erst noch durch spektroskopische Untersuchung darnach zu fahnden. Nachdem aber einmal von Pschorr nicht etwa als Vermutung, sondern als eine keiner Widerlegung mehr fähige Tatsache hingestellt worden war, daß die von mir an Hunden beobachtete narko* tische Wirkung lediglich auf Methämoglobinbildung zurückzuführen sei, stellte ich noch einige Versuche an, in denen ich nach intravenöser Applikation von Epiosin das Blut des lebenden Tieres mittelst des Spektroskops auf Methämoglobin durchforschte. Es geschah dies aber keineswegs in der Meinung, als ob eine so geringe

1) Vahlen, Über Morphigenin und Epiosin. Bericht der deutschen chem. Gesellschaft. XXXV. (1902.) 3045. 
Menge von Methämoglobin im Blute eines Tieres, die erst des Spektroskopes bedarf, um ihre Anwesenbeit zu verraten, auch nur im allerentferntesten für eine narkotische Wirkung verantwortlich gemacht werden könne. Nun aber zeigten drei derartig angestellte Versuche auch für die spektroskopische Wahrnehmung nicht die geringste Spur von Methämoglobin in den untersuchten Blutproben. Auf zwei dieser Versuche habe ich schon in der Erwiderung ${ }^{1}$ ) auf Pschorrs ersten Angriff mit einigen Worten hingewiesen. Sie mögen aber hier, zugleich mit einem dritten, ausführlich mitgeteilt werden.

\section{Versuch Nr. XII.}

Hund, Pintscher, $6 \mathrm{~kg}$ schwer. Eine Kanüle in die linke Jugularvene, eine andere in die rechte Karotis eingebunden. Die erste diente zur Injektion der Epiosinlösung, die zweite zur Entnahme der Blutproben, die spektroskopisch geprüft werden sollten.

$10 \mathrm{~h} .15 \mathrm{~m}$. Injektion von $2 \mathrm{ccm}$ einer 10 proz. Epiosinchloridlösung $(=0,2$ Epiosinchlorid).

$10 \mathrm{~h} .50 \mathrm{~m}$. Dentliche Narkose. Entnahme der ersten Blntprobe. Sie zeigt normale Farbe. Mit dem Spektroskop kann man keinen Methämoglobinstreifen wahrnehmen.

11 h. - m. Entnahme der zweiten Blutprobe. Verhält sich genau wie die erste.

$11 \mathrm{~h} .30 \mathrm{~m}$. Dritte Blutprobe. Verhält sich genau wie die vorige.

12 h. - m. Vierte Blutprobe. Verhält sich genan wie die vorige.

$12 \mathrm{~h} .10 \mathrm{~m}$. Tier wird verbluten gelassen. Das Blut zeigt die normale Farbe. Bei spektroskopischer Untersuchung kann man nicht die leiseste Andeutung eimes Methämoglobinstreifens wahrnehmen.

Der Sektionsbefund bietet nichts von einer schweren Organerkrankung, gesehweige denn anch nur die geringste Spur jenes bekannten Bildes, das man nach Vergiftung mit chlorsaurem Kalium und anderen methämoglobinbildenden Giften zu sehen bekommt.

\section{Versuch Nr. XIII.}

Sehr empfindlicher Hund, $5650 \mathrm{~g}$ schwer. In die Jugularvene der einen und die Karotis der anderen Seite je eine Kanäle eingebunden.

$10 \mathrm{~h} .50 \mathrm{~m}$. Injektion von $2 \mathrm{ccm}$ einer 10 proz. Epiosinchloridlösung (=0,2 Epiosinchlorid).

$11 \mathrm{~b} .-\mathrm{m}$. Narkose deutlich. Man kann durch Nase und Ohren Nadeln hindurchstechen, an die Ohren den Nadelhalter anklemmen, ohne daß eine Reaktion von seiten des Tieres erfolgt.

Erste Blutprobe entnommen. Normale Farbe. Mit dem Spektroskop kein Methämoglobinstreifen wahrzunehmen.

1) Über Morphigenin und Epiosin. Bericht der deutschen chem. Gesellschaft. XXXV. (1902.) 3017. 
$11 \mathrm{~h} .03 \mathrm{~m}$. Noch $1 \mathrm{ccm}$ der Epiosinlösung injiziert $(=0,1$ Epiosin. chlorid), das Tier fängt darauf an zu zittern.

11 h. $10 \mathrm{~m}$. Zweite Blutprobe. Normale Farbe. Mit dem Spektroskop kein Methämoglobinstreifen wahrzunelumen.

11 h. $20 \mathrm{~m}$. Noch $1 / 2 \mathrm{ccm}$ injiziert $(=0,05$ Epiosinchlorid $)$. Während der Injektion läßt das Tier Kot.

11 h. $25 \mathrm{~m}$. Dritte Blutprobe. Normale Farbe. Mit dem Spektroskop kein Methämoglobinstreifen zu sehen.

11 h. $40 \mathrm{~m}$. Noch $2 \mathrm{ccm}$ injiziert (=0,2 Epiosinchlorid).

12 h. 10 m. Vierte Blutprobe. Normale Farbe. Mit dem Spektroskop kein Methämoglobinstreifen zu sehen.

Beschreibung der Narkose: Man kann Nadeln durch Ohren und Nase stechen, Nadelhalter an ein Ohr klemmen, ohne daß der geringste Laut oder die geringste Bewegung erfolgt. Augen geschlossen.

12 h. $35 \mathrm{~m}$. Fünfte Blutprobe. Normale Farbe. Mit dem Spektroskop kein Methämoglobinstreifen zu erkennen. Das 'Tier wurde nun verbluten gelassen. Das Blut zeigte normale Farbe und bei spektroskopischer Prüfung keinen Methämoglobinstreifen. Die Sektion ergab nichts, was auf die Wirksamkeit eines methämoglobinbildenden Giftes schließen Iassen, überhaupt nichts, was auf eine schwere Organerkrankung hätte deuten können.

Versuch Nr. XIV.

Graues Kaninchen, $2300 \mathrm{~g}$ schwer. Es werden ihm $10 \mathrm{ccm}$ einer 10 proz. Lösung Epiosinchloridlösung ( $=0,2$ Epiosinchlorid) intravenös appliziert. Es tritt ein deutlich narkotischer Zustand ein. Nach 15 Minuten wird das Tier getötet. Im Blat, das ganz normale Farbe hat, läßt sich bei spektroskopischer Untersuchung kein Methämoglobinstreifen entdecken. Die Sektion ergibt keine mit bloßem Auge wabrnehmbare Veränderung der Organe.

Außer den früher mitgeteilten Versuchen an Hunden, die Pschorr bekannt sein multen, habe ich noch weitere Experimente an Hunden und Kaninchen ausgeführt, um die Wirkung des Epiosins auf Blutdruck und Atmung zu studieren. Auch in diesen Versuchen, die in Abschnitt I und II dieser Arbeit referiert sind, wurde nie eine Veränderung der Blutfarbe wahrgenommen, die nur den geringsten Verdaclit auf die Anwesenheit von Methämoglobin hätte rechtfertigen können.

Die oben gestellten drei Fragen muß ich demach auf Grund ron zahlreichen eigenen Versuchen mit nein beantworten.

Und wie stellt sich nun Pschorr zu diesen drei Fragen? Pschorr beantwortet diese drei Fragen überhaupt nicht.

Seinen ersten Angriff gegen meine Versuohe hat Pschorr aus- 
gesprochen, ohne ein einziges Tierexperiment anzuführen. Auf meine kurze Zurückweisung erfolgte keine Antwort. Aber acht Monate nach jenem ersten Angriff sandte Pschorr der Redaktion der Zeitschrift für physiologische Chemie l) eine in Gemeinschaft mit Bergell ausgefübrte Arbeit ein, in der die Anschauung, das Epiosin wirke lediglich infolge von Methämoglobinbildung narkotiseh, von neuem ausgesprochen wird, wobei im ganzen sechs mit Epiosin angestellte Tierversnche mitgeteilt werden.

Und welcher Art sind nun diese Versuche? Psehorr und Bergell teilen keinen einzigen Versuch an Hund oder Kaninchen mit.

Ich betrachte diese Nichtanfubrung irgend eines Epiosinversuches am Hund oder Kaninchen ohne ausdrückliche Angabeder Grüde, weshalb solcheVersuche unterblieben, alsvollgültigen Beweis dafür, daß Pschorr und Bergell nicht imstande waren, meine an diesen Tieren gemachten Beobachtungen als irrtimlich zu erweisen.

Meine Auseinandersetzung mit Psehorr hat damit prinzipiell ilıre Erledigung gefunden.

Wenn ich gleichwohl noch weiter auf das, was Pschorr für eine experimentelle Begründung seiner Auffassung der Epiosinwirkung auszugeben sich nicht scheut, in aller Kürze eingehe, so geschieht dies, wie ich ausdrücklich vorausschicke, keineswegs deshalb, weil ich seiner Beweisfuhrung anch nur den geringsten Wert beimesse.

Wie bereits oben gesagt, fihren Pschorr und Bergell im ganzen sechs Epiosinversuche an Tieren an. Davon sind zwei Versuche an Fröschen angestellt, an denen sie, ebenso wie ich, eine narkotische Wirkung beobachteten und gleichwohl im Blute spektroskopisch nur Oxyhämoglobin, aber keine Spur von Methämoglobin nachweisen konnten. Also: Pschorr und Bergell konnten meine Froschversuche mit Epiosin nur bestätigen ${ }^{2}$, bielten es aber nicht für notwendig, dies a usdrucklich auszu. sprechen.

Die ẗbrigen vier Versuche ron Psehorr und Bergell sind an "Warmblütern" ausgeführt, nämlich an einer Mans und drei Vögeln. Der Tenor ihrer Beweisfuhruug ist nun folgen-

1) P. Bergell und R. Pschorr, Über die physiologische Wirkung einiger Phenanthrenderivate. Zeitschr. f. physiol. Chemie. XXXVIII. (1903.) 16.

2) L. C. S. 35 t. 36 . Vers. Nr, XXXV und XXXVIII. 
der: Dawir nicht imstande gewesen sind, im lebenden Hund oder Kaninchen nach Epiosininjektion Methämoglobinbildung nachzuweisen, müssen wir irgend ein Tier aufzutreiben suchen, bei denendies doch vielleicht noch gelingt. Gläckt dies wirklich, dann ist es offenbar, daß auch die am Hund und Kaninchen $z u$ beobachtende narkotische Wirkung keine morphiumähnliche war, sondern auf Methämoglobinbildung beruhte! Bedarf diese Art der Beweisführung noch eines Kommentares? Muß noch ausdrücklich gesagt werden, daß irgendein narkotischer Zustand (gleichgültig ob man ihn morphiumäbnlich nennen will oder nicht), in dem sich ein Tier befindet, wenn man ihn schon auf Methämoglobinbildung zurtickführen will, diese Methämoglobinbildung doch wenigstens in demselben Organismus stattfinden muß, dessen narkotischer Zustand damit erklärt werden soll? Oder sollte es irgend jemand für möglich halten, daß ein Hund oder Kaninchen in einen narkotischen Zustand versetzt werden könnte durch Methämoglobin, das sich in einem Hahn oder sonst einem Vogel vorfindet.

IV. Wirkung des Epiosins auf Mäuse.

Ich schicke zunächst das Protokoll des Mauseversuches von Pschorr und Bergell11) voraus.

Maus. Zweimal 0,1 g Epiosin. 14 Minuten nach der ersten subkutanen Injektion (10 proz. Lösung) noch keine deutliche Wirkung. Zweite Injektion 4 Minuten darauf, Bewegungen matter. Schmerzempfindung herabgesetzt. Stärkere Reize lösen prompte Reflexe aus. Die Herabsetzung der Reflexerregbarkeit ist eine weit geringere als bei Pheranthrenchinonsulfosäuren. Nach 6 Minuten kriecht das Tier langsam unter Nachziehen der hinteren Extremitäten. Nach $14 \mathrm{Mi}$ nuten läßt es sich auf den Rücken legen. Zittern und klonische Zuckungen. Getötet: Herzblut ergibt Methämoglobinämie deutlich.

Was mir bei der Sektion der Versuchsbeschreibung besonders auffiel, war der Mangel einer genaueren Angabe uber die Art und Weise, wie das Methämoglobin im Blute der Maus nachgewiesen wurde. Da die Autoren nichts weiter aussagen, als daß sie das Methämoglobin im Herzblut gefunden hätten, so darf man woh] schließen, daß diese Feststellung mit dem Spektroskop gemacht worden ist, denn hätte die geöffnete Mauseleiche sofort jene für das bloße Auge wahrnehmbare, charakteristisch braune Farbe des Blutes

1) L. c. S. 35 . Vers. Nr: XXXVI. 
und der Organe dargeboten, die niemandem mehr aus dem Gedächtnis schwindet, der je ein mit chlorsaurem Kalium (oder einem ähnlich auf das Blut wirkenden Stoffe) vergiftetes Tier obduziert hat, so hätten die beiden Experimentatoren gewiß nicht versäumt, es nicht nur anzuführen, sondern sogar in gesperrten Lettern drucken zu lassen.

Darin aber, daß bei der ungeheuren Dosis von Epiosin, mit der das kleine Tier geradezu angefüllt worden war, doch nur eine so geringe, erst mit dem Spektroskop nachweisbare Menge von Methämoglobin im Blute gefunden werden konnte, lag für mich das Hauptinteresse dieses Versuches, das mich veranlaßte, ihn zu wiederholen. Denn man iberlege sich die Konzentration von Blut und Epiosinlösung, die in dem Mäuseversuch von Pschorr und Bergell aufeinander einwirkten. Sie haben selbst das Gewicht ibrer Maus nicht angegeben. Ich nehme daher das Mittel aus den Gewichten der vier weißen Mäuse, die zu meinen eigenen Versuchen verwendet wurden. Dieses Mittel beträgt 15,52 g. Daraus (durch Division mit 13) berechnet sich für die Blutmenge einer Maus $=1,2 \mathrm{~g}$. Und auf diese Blutmenge ließen Psch orr und Bergell $2 \mathrm{ccm}$, also beinahe die doppelte Menge einer 10proz. Epiosinlösung einwirken. Sind das nicht Konzentrationsverhältnisse, wie man sie selbst im plumpsten Reagensglasversuche kaum erreichen kann? Noch schlagender wird dies, wenn wir die applizierte Menge des Epiosins auf die Gesamtmenge des Blutfarbstoffes berechnen, die das kleine Tierchen besessen haben mochte. Nehmen wir an, daß das Mänseblut (genaue Bestimmungen liegen darüber nicht vor) etwa 10 Proz. Blutfarbstoff enthält, so verfügt eine Maus im Durehschnitt über $0,1 \mathrm{~g}$ Blutfarbstoff. Pschorr und Bergell haben demnach ihrer Maus doppelt soviel Epiosinchlorid injiziert als sie Blutfarbstoff besaß. Sollte man, da ja das Epiosin in der Tat im Reageusglase Methämoglobinbildung veranlaßt, trotzdem es bekannt ist, daß daraus für eine gleiche Wirkung im lebenden Organismus nichts gefolgert werden darf, nicht doch bei einem derart angestellten Tierversuche die Bildung von Methämoglobin für wahrscheinlicher halten als das Gegenteil?

Wollte man, was bei der bekannten Differenz im Verhalten verschiedener Tiere gegen ein und dasselbe Gift ganz verkehrt wäre, von einem einzigen Versuch an einer Maus Schlüsse auf den Hund ziehen, so ist die allererste Voraussetzung für ein solehes Verfahren die, daß die Versuchsbedingungen wenigstens einigermaßen äquivalente sind. $\mathrm{Zu}$ den Versuchsbedingungen gehört aber im pharmakologischen Experiment in erster Linie die Dosis 
der angewandten Substanz. Berechnet man die von Pschorr und Bergell der Maus injizierte Epiosinmenge auf den Hund, als Durchschnittsgewicht für diesen $7 \mathrm{~kg}$ angenommen, so finden wir die ungeheure Zahl von $40 \mathrm{~g}$ Epiosinchlorid! Meine Hunde erhielten dagegen nur einige Dezigramme. Diese Zahlen reden eine deutliche Sprache. Da ich einmal beim Rechnen bin, so mag der Vergleich bis zum Mensehen fortgeführt werden. Bei einem Durchschnittsgewicht von nur $65 \mathrm{~kg}$ berechnet sich aus dem Mäuseversuch von Pschorr und Bergell für den Menschen die ungenre Dosis von $371 \mathrm{~g}$ Epiosinchlorid. Was eine solche kolossale Menge von Epiosin (auf einmal einverleibt, wenn dies überhaupt möglich wäre) im mensehlichen Organismus für Wirkungen entfalten mag, vielleicht auch die Bildung von gerade noch mit dem Spektroskop nachweisbaren Spuren von Methämoglobin - daräber Betrachtungen oder Experimente anzustellen, hat doch weder theoretiscbes noch praktisches Interesse.

Ich habe im ganzen vier Versuche an Mäusen ausgefülırt, nicht mehr und nicht weniger, und zwar, worauf ich sonst freilich nie verfallen wäre, nach dem Vorgange von Pschorr unter Anwendung ganz ungeheuerlicher Epiosindosen. Meine Versuche an Mäusen hatten alle dasselbe Ergebnis.

Versuch Nr. XV.

Weiße Maus, 16,45 g schwer.

$11 \mathrm{~h}$. - m. Injektion von $1 \mathrm{ccm}$ einer 10 proz. Lösung von Epiosinchlorid ( $=0,1$ Epiosinchlorid) unter die Rückenhaut.

11 h. $10 \mathrm{~m}$. Sitzt ruhig da, die Nase auf den Boden des Glases geduckt. Ist ganz entschieden nicht mehr so lebhaft wie vorher. Mit einer Nadel in den Schwanz oder eine Pfote gestochen, zuckt sie zusammen. Aber die Bewegungen sind dabei ungeschickter als zuvor. Einen gewissen Grad von Benommenheit wird man nicht leicht in Abrede stellen können.

11 h. $20 \mathrm{~m}$. Bewegt sich nur schwer und langsam vorwärts. Zieht man sie mit einer Pinzette an einem Ohr in die Höhe, so zappelt sie kaum.

$11 \mathrm{~h} .30 \mathrm{~m}$. Ich nehme jetzt die Maus aus dem Glase und setze sie frei auf den Tisch, ohne Sorge, daß sie mir entschlüpfen könnte. Sie läuft etwa zwei Körperlängen weit, dann bleibt sie stehen. Heftig in den Schwanz gekniffen, kriecht sie ungeschickt weiter, nicht mit den hastigen Bewegungen, wie man sie an Mäusen zu sehen gewohnt ist, sondern in der phlegmatischen Gangart einer Schildkröte. Sie bleibt dann nach kurzer Wegestrecke stehen, die Schnauze auf die Tischplatte gelegt, den Schwanz lang ausgestreckt. Die Ohrgefäße mit der Lupe betrachtet, zeigen noch deutlich rote Farbe. Auch der Augenhintergrund blitzt noch ebenso rot wie vor dem Versuch. 
$11 \mathrm{~h} .40 \mathrm{~m}$. Sitzt noch an demselben Fleck regungslos da.

11 h. $45 \mathrm{~m}$. Wird auf den Rücken gelegt und bleibt liegen. Es beginnen schwache, klonische Zuckungen der Extremitäten. Die Ohrgefäße, mit der Lupe betrachtet, zeigen noch immer die normale rote Farbe. Ebenso verhält sich die Zunge. Auch die Augen funkeln ebenso rot wie vorher.

$11 \mathrm{~h} .55 \mathrm{~m}$. Liegt noch ebenso da. Wird, um sie vor Abkühlung zu schützen, auf den Rücken zwischen zwei Wattebäusche gelegt, wo sie ruhig liegen bleibt. Klonische Zuckungen jetzt schwächer. Lid- und Kornealreflex erhalten, aber sehr schwach. Man kann mit dem Nadelhalter den Schwanz zermalmen, es erfolgt keine Reaktion. An der Stelle der Verwundung tritt ein Blutstropfen aus, der die normale hellrote Farbe des Arterienblutes zeigt.

1 h. - m. Das Tier befindet sich noch in demselben Zustand wie vorher. Es wird jetzt der Thorax mit einem Scherenschnitt geöffnet, das Herz abgeschnitten und samt Blut in einem Reagensglas aufgefangen. Die spektroskopische Untersuchung ergab die vollkommene Abwesenheit eines Methämoglobinstreifens. Die Blatprobe im Reagensglase blieb nicht nur einige Standen, sondern bis zum nächsten Tage stehen. Auch dann konnte mit dem Spektroskop kein Methämoglobin entdeckt werden.

Nach der Entnabme des Herzens warde die Maus geköpft und der Leib vollkommen geöffinet. Das Blut, sowie sämtliche Organe zeigten normale Farbe, jedenfalls nicht die geringste Bräunung, die auf Anwesenheit von Methämoglobin deutete.

\section{Versuch Nr. XVI.}

Weiße Maus, 15,3 g schwer.

$4 \mathrm{~h} .10 \mathrm{~m}$. Injektion von $1 \mathrm{ccm}$ einer 10 proz. Lösung von Epiosinchlorid $(=0,1$ Epiosinchlorid) unter die Rückenhaut.

$4 \mathrm{~h} .15 \mathrm{~m}$. Sitzt mit halbgeschlossenen Augen da, die Nase auf den Boden des Glases gedräckt. Beim Anstoßen, Anfassen mit Pinzette erfolgen träge Bewegungen. Es besteht also schon der Beginn einer parkotischen Wirkung.

4 h. $30 \mathrm{~m}$. Wird jetzt ans dem Glase genommen und frei auf den Tisch gesetzt. In den Schwanz gekniffen, kriecht das Tier langsam vorwärts, bleibt aber bald stehen.

$4 \mathrm{~h} .45 \mathrm{~m}$. Kann auf den Rücken gelegt werden und bleibt liegen. Es treten schwache klonische Zuckungen an den vorderen Extremitäten auf. Wiederholt in den Schwanz gekniffen, erfolgt entweder nur eine schwache oder gar keine Reaktion. Wird in Watte gepackt und bleibt so liegen bis

5 h. $59 \mathrm{~m}$. Es wird, wie im vorigen Versuch, Herz samt Blut entnommen. Die spektroskopische Untersuchung ergab vollkommene Abwesenheit von Methämoglobin. Auch am nächsten Tage konnte in der Blutprobe mit dem Spektroskop kein Methämoglobinstreifen entdeckt werden.

Nach Heransnahme des Herzens warde der Kopf abgeschnitten und 
das Tier obduziert. Blut und Organe zeigten keine Farbenveränderung, die auf Anwesenheit von Methämoglobin hätte deuten können.

In den beiden beschriebenen Versuchen erhielten die Mäuse 0,1 Epiosinchlorid, also die Hälfte der von Pschorr und Bergell ihrer Maus applizierten Dose. chlorid.

In den beiden folgenden Versuchen erhielten die Mäuse 0,2 Epiosin-

Versuch Nr. XVII.

Weiße Maus, $14,15 \mathrm{~g}$ schwer.

$10 \mathrm{~h} .40 \mathrm{~m}$. Injektion von $1 \mathrm{ccm}$ einer 10 proz. Epiosinchloridlösung (=0,1 Epiosinchlorid) unter die Rückenhaut.

$11 \mathrm{~b} . \quad-\mathrm{m}$. Sitzt mit niedergeduckter Nase da. Ist garnicht mehr schreckhaft. Nähert man der Maus einen Gegenstand oder berïhrt sie, so entschließt sie sich nur sehr langsam, die Flucht zu ergreifen.

$11 \mathrm{~h} .05 \mathrm{~m}$. Wird jetzt frei auf die Tischplatte gesetzt. Es besteht keine Befürchtung, daß sie entwisehen könnte.

$11 \mathrm{~b} .20 \mathrm{~m}$. Liegt in Watte verpackt auf dem Rücken. Sticht man mit Nadeln in den Schwanz, so erfolgt eine ganz schwache Zuckung. Das Tier bleibt aber ruhig liegen. Augen halb geschlossen. Zunge und Ohrgefäße schön rot. Ebenso Augenhintergrund.

Klonische Zuckungen werden bei diesem Tiere garnicht beobachtet.

$11 \mathrm{~h} .45 \mathrm{~m}$. Noch $1 \mathrm{ccm}$ der $10 \mathrm{proz}$. Epiosinlösung injiziert. Hat jetzt im ganzen 0,2 Epiosinchlorid erhalten. Auch jetzt treten keine klonischen Zuckungen auf.

$12 \mathrm{~h}$. - m. Herz samt Blut wie in den vorigen Versuchen herausgenommen. Bei spektroskopischer Untersuchung zeigte sich nicht die geringste Andentung eines Methämoglobinstreifens, auch nach stundenlangem Stehender Blutprobe nicht.

Obduktionsbefund des geköpften Tieres wie in den vorhergehenden Versuchen.

\section{Versuch Nr. XVIII.}

Weiße Mass, 16,25 g sehwer.

Bekam je 0,1 Epiosinchlorid (in 10 proz. wässeriger Lösung) unter die Rïckenhaut injiziert und zwar zweimal innerhalb eines Zeitraumes von 18 Minuten, genau wie in dem Versuch von Pschorr und Bergell. Die Wirkung war im großen und ganzen dieselbe wie bei den anderen Mäusen. Doch soll nicht verschwiegen werden, daß die narkotische Wirkung in diesem Falle keinen so hohen Grad wie in den übrigen Experimenten erreichte, wahrscheinlich infolge der kürzeren Dauer des Versuches. Aber natürlich konnte das Tier ebenfalls frei auf die Tischplatte gesetzt werden, obne daß ein Entwischen zu befürchten war.

5 h. $25 \mathrm{~m}$. Erste Injektion von 0,1 Epiosinchlorid.

$5 \mathrm{~h} .43 \mathrm{~m}$. Zweite Injektion von 0,1 Epiosinchlorid.

5 h. $58 \mathrm{~m}$. Entnahme des Herzens samt Blut wie in den übrigen Versuchen. In der Blutprobe ist mit dem Spektroskop kein Methämoglobinstreifen zu entdecken. Auch nach stundenlangem Stehen nicht. Die Obduktion des geköpften Tieres 
läßt bei dem Anblick des Blutes und der Organe nicht den geringsten Verdacht auf Anwesenheit von Methämoglobin a ufkommen ${ }^{1}$ ).

Bei dem diametralen Gegensatz, in dem das Ergebnis meiner vier Mäuseversuche zu dem des einen Versuches von Pschorr und Bergell sich befindet, wäre es sehr wünschenswert gewesen, zu wissen, wie viele Versuche diese beiden Autoren im ganzen angestellt haben und wie viele davon ein anderes Resultat ergeben hatten, als dieser eine, den sie der Mitteilung für wert erachtet haben. Daß es sich aber in dem Befund dieses allein dastehenden Versuches von Pschorr und Bergell im besten Falle nur um ein vereinzeltes Vorkommnis handeln kann, das (um von einer Übertragung auf andere Tiere ganz und gar zu schweigen) selbst in einer Versuchsanordnung wie der angewandten, geschweige denn bei einer solchen, wie sie allein für pharmakologische Deduktionen in Anwendung kommen dürte, keine Verallgemeinerung der Epiosinwirkung selbst nur für Mäuse zulassen würde, scheint mir auf der Hand zu liegen.

Nun bin ich aber überdies imstande zu zeigen, daß Pschorr und Bergell wahrscheinlich das Opfer eines groben Beobachtungsfehlers geworden sind. Ich hatte nämlich an die Möglichkeit gedacht, dab bei diesen riesengroßen Epiosindosen in dem Kadaver der Mäuse noch post mortem eine Methämoglobinbildung eintreten und dies zu Täuschungen Anlaß bieten könnte. Es wurden daher die Mäuseleichen noch eine Weile, bis etwa eine Stunde nach der Tötung, liegen gelassen, und dann die Färbung von Blut und Organen festgestellt. Dabei fand sich in keinem Falle eine mit bloßem Auge wahrnehmbare (spektroskopische Untersuchung wurde nicht vorgenommen) Veränderung, die zur Vermutung auf Anwesenheit von Methämoglobin berechtigt hätte.

Allein im dritten Mäuseexperiment (Versuch Nr. 5) wurde folgende interessante Wahrnebmung gemacht. Als ich nämlich den in einer Porzellanschale liegenden Mäusekadaver nach einiger Zeit wieder betrachtete, hatten einige Blutstropfen, die bei der Öffnung und der Köpfung des Tieres an den Rand der Schale gespritzt waren, noch die unveränderte hellrote Farbe. Dagegen zeigte eine kleine Blutlache, die den Rücken des Tieres berührte, jene braune

1) Herr Geheimrat Harnack und mein Kollege am hiesigen Institut, Herr Dr. Fuld, nahmen auf meine Einladung mit liebenswürdigstem Interesse an meinen sämtlichen Versuchen mit Mäusen teil. Beide überzeugten sich von dem frappanten Widerspruch, den meine Feststellungen zu den Angaben von Pschorr und Bergell darboten. 
Farbe, die schon dem unbewaffneten Auge die Anwesenheit von Methämoglobin verrät. Die Ursache dieser Erscheinung wurde alsbald offenbar, als ich den Körper des Tieres umwandte. Dabei konnte man nämlich feststellen, daß von dem Scherenschnitt, der den Kopf rom Rumpfe getrennt hatte, auch die ausgedehnte Infiltration getroffen worden war, die von der Injektionsstelle aus, was bei der unerhört großen Menge der Giftlösung nicht zu verwundern, in weitem Umkreise sich unter der Ruckenhaut ausgebreitet hatte. Man sah nun aus der sulzig infiltrierten Sehnittfläche von dem vermutlich noch recht beträchtlichen Rest der nicht vollkommen resorbierten Injektionsflissigkeit Tropfen für Tropfen heraustreten und sich mit dem an der Wundfläche von Kopf und Hals hervorsickerndem Blute vermischen. Auf diese Weise kam nicht nur eine postmortale, sondern auch eine extravasculäre Reaktion des Epiosins auf das Blut zustande und zwar unter Konzentrationsverhältnissen, wie sie kaum der roheste Reagensglasversuch vor Augen führen konnte.

Es ist nun leicht zu begreifen, wie man auf diese Weise auch im Blute lebender Hunde (oder Kaninchen) die Anwesenheit von Methämoglobin ganz leicht demonstrieren könnte. Man braucht nur dafür zu sorgen, auf einer Wundfläche, wie man sie z. B. beim Bloßlegen eines Gefäßes zur Injektion oder zur Anstellung eines Blutdruckversuches vor sich hat, von einer konzentrierten Epiosinlösung eine hinreichend große Menge darauf fließen zu lassen, was bei einiger Ungeschicklichkeit auch ganz absichtslos geschehen könnte, und man würde dann vor seinen Augen, auch ohne erst das Spektroskop zu Hilfe nehmen zu müssen, die schönste Methämoglobinreaktion sich vollziehen sehen. Aber freilich wäre diese Methämoglobinbildung nicht innerhalb des kreisenden Blutes vor sich gegangen und man wäre nicht berechtigt, sie als Ursache irgendeiner Allgemeinwirkung des Epiosins anzusehen.

Was man aus diesen nach Pschorr und Bergells zwar nicht als mustergiltig zu bezeichnenden Methodik an Mäusen angestellten Versuchen lernen kann, ist nicht neu, durfte aber bisher noch nicht in so verbliffender Deutlichkeit demonstriert worden sein. Nämlich: Ein im Reagensglase den Blutfarbstoff unter Methämoglobinbildung zersetzendes Gift kann sieh innerhalb des kreisenden Bluteseines lebenden Organismus vollkommen indifferent gegen den Blutfarbstoff verhalten, selbst wenn die injizierte Gift- 
Die chemische Konstitution des Morphins in ihrer Beziehung zur Wirkung. 151

menge doppelt so grob ist als die zur Verfügung stehende Gesamtmenge des Blutfarbstoffes.

\section{Wirkung des Epiosins auf Vögel.}

Von den vier Versuchen an "Warmblütern", die Pscborr und Bergell im ganzen in der Lage sind als Stütze ihrer Anschauung beizubringen, die von mir an Hunden (und Kaninchen) experimentell bewiesene narkotische Wirkung des Epiosins berube lediglich auf einer Methämoglobinbildung, bleiben nach Britik des äußerst interessanten Mäuseversuches noch drei übrig. Diese drei "Warmblater"-Versuche sind alle an Vögeln angestellt.

Einer dieser Versuche an Vögeln ist an einer Taube l) ausgefiuhrt. Nach subkutaner Injektion von 0,1 Epiosinchlorid in 10 proz. Lösung stellten sich dentliche Zeichen einer narkotischen Wirkung ein, mühsame Bewegungen, Störungen des Gleichgewichts usw. Dabei traten aber auch noch andere Symptome auf, Erbrechen und blutige Defäkation. Nach 70 Minuten hatte sich das Tier etwas erholt, befand sich aber noch an den folgenden Tagen in einem krankhaften Zustand. Am dritten Tage war die Taube tot. Den Schluß der Versuchsbeschreibung bildet die kurze Mitteilung: "Methämoglobinämie leicht nachweisbar". Da über die Art, wie dieser Nachweis geschah, nichts ausgesagt wird, dürfte die Blutveränderung wohl nur mit dem Spektroskop erkennbar, also jedenfalls nicht sehr hochgradig gewesen sein.

Da die Autoren ferner nicht mitteilen, wie viele Taubenversuche sie mit Epiosin tiberhaupt angestellt haben, und in wie vielen dabei Methämoglobinbildung beobachtet werden konnte und in wie vielen nicht, scheint mir die Vermutung nicht allzu kỉhn, der angeführte Taubenversuch möchte, was die Metbämoglobinbildung betrifft, der* einzige in seiner Art gewesen sein.

Als SchluBstein ihres experimentellen Beweismaterials fubren Pschorr und Bergell zwei Versuche an Hähnen ${ }^{2}$ ) an, die ich im folgenden wörtlich wiedergeben will.

Erster Hahnversuch von Pschorr und Bergell ${ }^{3}$ ).

Hahn. 0,2 Epiosinchlorid, subkntan. Junges Tier. Kamm rot. 25 Minuten p. i. Kamm dunkler, Tier müder, läuft langsamer. Sensibilität erhalten. 35 Minuten p. i. Kamm dunkelblau. Tier sitzt still, scheint

1) L. c. S. 36. Vers. Nr. XXXVII.

2) L. c. S. 36. Vers. Nr. XL und LVI.

3) L. c. S. 36 . Vers. Nr, XL. 
schläfrig. Reflexerregharkeit herabgesetzt. Keine ausgesprochene anal. getische Wirknng. Kammschnittblut: Methämoglobin.

Später Defäkation, einmal blutig. Freßunlust. Der leicht soporöse Zustand dauert tagelang an. Tier bleibt am Leben.

Zweiter Hahnversuch von Pschorr und Bergell 1).

Ein mit $0,2 \mathrm{~g}$ salzsaures Epiosin subkutan vergifteter Hahn zeigt noch nach 4 Tagen Verfärbung des Kammes. Einige Tropfen des Kammschnittblutes zeigen Methämoglobinämie spektroskopisch.

Ohne mich in eine nähere Besprechung dieser beiden Versuche einzulassen, begnüge ich mich damit, meine eigenen Beobachtungen an cinem mit Epiosin vergifteten. Hahn mitzuteilen.

Versuch Nr. XIX.

Junger Hahn mit prachtvollem rotem, aufrechtstehendem, glänzendem Kamm.

5 h. $05 \mathrm{~m}$. Subkutane Injektion von 0,2 Epiosinlösung in 10 proz. Lösung.

$5 \mathrm{~h} .35 \mathrm{~m}$. Noch immer keine Wirkung walırzunehmen. Vielleicht ist das Tier etwas ruhiger und etwas leichter zu ergreifen, als vor der Injektion. Ich möchte mich aber nicht getrauen, mit Bestimmtheit von einer narkotischen Wirkung zu sprechen ${ }^{2}$ ). Im übrigen ist am Tier kein Symptom, rein gar keines wahrzunehmen. Der Kamm erstrahlt in prachtvollem Rot wie vorher. Eine Probe des durch einen Messerschnitt in den Kamm gewomnenen Blutes ist schön rot gefärbt und läßt mit dem Spektroskop nicht die geringste Spur eines Methämoglobinstreifens erkennen ${ }^{3}$ ).

$6 \mathrm{~h} .30 \mathrm{~m}$. Hahn ebenso munter wie vorher. Kamm prachtvoll rot, ohne die geringste Veränderung. In einer $z$ weiten Blutprobe, dem Kamm durch einen Messerschnitt entnommen, kann mit dem Spektroskop kein Methämoglobinstreifen wahrgenommen werden.

Den ganzen Nachmittag und Abend befindet sich das Tier munter, ebenso die folgenden Tage. Sein Kamm erfreut den Beschaner stets von neuem durch seine ganz besonders auffallend glänzendrote Farbe. Auch im ubrigen ist nicht das geringste Vergiftungssymptom wahrzunehmen.

Ohne in den Fehler zu verfallen, aus einem einzigen Versuch am Hahn alles erscbließen zu wollen, was Epiosin in dem Organismus

1) L. c. S. 36 . Vers. LVI.

2) Daß die angewandte Dosis des Epiosins sich in dieser Hinsicht so gut wie vollkommen wirkungslos erwies, konnte mich bei der bekannten Renitenz der Hähne gegen die narkotische Wirkung des Morphins und der, wie aus den Versuchen an Hunden zu ersehen, geringeren narkotischen Kraft des Epiosins, nicht im geringsten überraschen.

3) Ebensowenig wie mir gelang es Herrn Geheimrat Harnack und Herrn Dr. Fuld, in dieser wie in der zweiten Blatprobe mit dem Spektroskop einen Methämoglobinstreifen wahrzunebmen. 
dieses Vogels eventuell einmal zu vollbringen vermag, so kann das doch wenigstens mit voller Bestimmtheit ausgesagt werden, daß das Epiosin in der angewandten Dosis für Hähne eine sehr harmlose Substanz ist und jedenfalls kein heftiges Blutgift, das durch Methämoglobinbildung schwere Vergiftungssymptome hervorzurufen imstande ist.

Meine Beobachtungen stehen im schroffen Gegensatz zu den Angaben von Pschorr und Bergell. Während ich aber die abweichenden Ergebnisse dieser Autoren bei den Mäuseversuchen auf einen schweren Beobachtungsfehler zurïckführen konnte, bin ich bei den Versuchen an Hähnen nicht in der gleichen Lage. Es bleibt mir nur eine Vermutung übrig, nämlich die, daß das von Pschorr und Bergell angewandte Epiosinpräparat nicht frei von Verunreinigungen war. Ich selbst habe mich von der Reinheit meines in den früher wie in den hier mitgeteilten Tierversuchen angewandten Epiosins überzeugt, was bei einer Substanz, die leicht kristallisiert und einen konstanten Schmelzpunkt besitzt, so außerordentlich einfach ist. Das Epiosin kann in verschiedener Weise synthetisch dargestellt werden. Wie Pschorr und Bergell es gewonnen und wie sie sich von seiner Reinheit überzeugt haben, geben sie nicht an. Und doch waren sie hierzu um so mehr verpflichtet, als sie aus den möglieherweise von den meinigen differierenden Beobachtungsresultaten heftige Vorwürfe gegen meine eigenen Angaben herzuleiten gedachten.

Ich bin am Schluß meiner Darstellung der bisher gefundenen Wirkungen des Epiosins und der teils theoretischen, teils experimentellen Kritik der Versuche von Pschorr und Bergell, die sich zu dem Ausspruch berechtigt fühlten ${ }^{1}$ ):

"Im vorliegenden Falle ist jedenfalls gerade beim Epiosin die von Vahlen tabersehene Methämoglobinämie in dem Maße vorhanden, daß sie zur Erklärung der geringfügigen narkotischen Wirkung allein ausreicht. Entgegen den Einwendungen von Vahlen, daß bei zwei in dieser Beziehung an einem Hunde und an einem Kaninchen mit je 0,2 Epiosinchlorhydrat angestellten Versuchen sich Methämoglobin nicht habe nachweisen lassen, müssen wir darauf hinweisen, daß dieser. Nachweis bei jedem unserer Fälle an Warmblütern ${ }^{1}$ ) gelang.

1) L. c. S. 31.

2) Eine Maus, eine Taube und zwei Hähne. 
Ich fasse dagegen die Summe meiner Beobachtungen und Überlegungen in dem Satz zusammen:

Pschorr und Bergell ist es nach achtmonatlichen Bemuhangen nicht gelungen, auch nur den leisesten Schimmer eines Beweises für die Behauptung, die narkotische Wirkung des Epiosins beruhe auf Methämoglobinbildung, zu erbringen.

VI. Ü̉ber" "Morphigeninsulfosäur e".

In meiner Arbeit: "Über die chemische Konstitution des Morphins usw." war der Ausgangspunkt vieler chemischer Experimente und Versuche an Tieren das salzsaure Salz des von mir zuerst dargestellten 9-Amino-10-0xyphenanthrens, das ich wegen seiner Beziehungen zu Substanzen mit morphiumähnlicher Wirkung als Morphigenin bezeichnet habe.

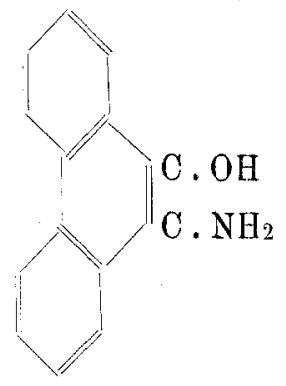

Durch Einwirkung von konzentrierter Schwefelsäure auf Morphigenin in verschiedenen relativen Mengenverhältnissen, bei versoliedenen Temperaturen und bei verschiedener Dauer der Einwirkung erhielt ich Produkte von zum Teil mehr oder weniger deutlich morphiumähnlicher Wirkung. Doch gelang es mir nicht, wie in meiner Arbeit auch ausdrücklich angegeben ist, die wirksamen Substanzen in reinem Zustand zu isolieren. Ich bezeichnete aber die zu den Tierversuchen angewandten Reaktionsprodukte mit aller Reserve als Morphigeninsulfosäuren.

Die diesbezïglichen Stellen meiner Abhandlung lauten:

Seite 386: "Ich versuchte daher durch Variation der angewandten Schwefelsäuremengen und der einwirkenden Temperatur beständigere Verbindungen zu erhalten. Aber zahllose Bemühungen nach dieser Richtung lieferten zwar Reaktionsprodukte von eigentümlicher Wirkung, aber in keinem Falle gelang es, die wirksame Substanz in reinem und unveränderlichem Zustande und konstanter Wirkung $z u$ isolieren." 
"Aus der großen Zahl diesbezüglicher Versuche sei eine Reihe mitgeteilt, in welchen weiter nicht individualisierbare Stoffe entstanden, welohe unzweideutige, morphiumähnliche Wirkung aufwiesen."

Und am Schlusse des ganzen Abschnittes, Seite 395, lautet es:

„Die Zahl der angeführten Versuche könnte durch Anführung weiterer Protokolle aus meinen Versuchsjournalen vergrößert werden. Sie wird aber genügen, um die Berechtigung des Sehlusses wahrscheinlich zu machen, daß durch Einwirkung von konzentrierter Schwefelsäure auf das Morphigeninchlorid Produkte von mehr oder weniger deutlich morphiumähnlicher Wirkung gebildet werden. Vermutlich handelt es sich um isomere Mono-Di-usw.-Sulfosäuren, die sowohl in der Qualität als auch in der Intensität ihrer Wirkung voneinander differieren und deren Trennung und Reindarstellung auf große Schwierigkeiten stößt."

Wie schon gesagt, waren die Sulfurierungsprodukte des Morphigenins in verschiedener Weise gewonnen worden und hatten dementsprechend auch verschiedene Eigenschaften. Entweder wurde die Sulfurierung vorgenommen dureh Erhitzen von Morphigenin mit der 5 fachen Menge konzentrierter Schwefelsäure auf $150-160^{\circ}$ oder mit der 6 fachen Menge konzentrierter Schwefelsäure auf $140^{\circ}$ oder bloß bei Wassertemperatur usw. Ebenso wechselte die Daner der Erhitzung. Auch die Isolierung der Sulfurierungsprodukte war in den einzelnen Fällen eine verschiedene. In den einen Versuchen waren sie in Wasser schwer löslich oder fast unlöslich. Sie konnten daher durch Waschen mit Wasser von dem größten Teil der Schwefelsäure befreit werden. In anderen Experimenten waren die zu den Tierversuehen dienenden Sulfurierungsprodukte in Wasser leicht löslich und wurden durch Sättigung mit Kochsalz isoliert. In einigen Fällen wurde der Kochsalzniederschlag wieder in Wasser gelöst und aus dieser Lösung durch Bleiessig ein unlösliches Bleisalz dargestellt, durch dessen Zerlegung mit Schwefelwasserstoff das wirksame Produkt gewonnen wurde. Auch die Barytsalze der erhaltenen Sulfurierungsprodukte waren in Wasser bald löslich, bald unlöslich.

Der Vorwurf, den Pschorr sowohl bei seinem ersten Angriff wie acht Monate später in der mit Bergell verfaßten Abhandlung meinen Studien über "Morphigeninsulfosäure" macht, bat eine rein chemische und eine pharmakologische Seite.

In ehemischer Hinsicht behauptet Pschorr mit der ihm eigentümlichen Kürze und Bündigkeit, was ich (mit aller Reserve) als 
"Morphigeninsulfosäure" bezeichnet babe, sei nichts anderes als Phenanthrenchinonsulfosäure gewesen.

Man sieht sich in seinen beiden Abhandlungen vergeblich nach einem Beweis für diese Behauptung um. Weder gibt er genau an, welche von meinen verschiedenen Sulfurierungsmethoden er angewandt, noch auf welche Weise er Phenanthrenchinonsulfosäure aus dem Reaktionsprodukt isoliert hat, wie er denn auch keine einzige Analyse ${ }^{2}$ ) anführt. In der Arbeit mit Bergell werden die zu den Tierversuchen verwandten Produkte einfach bezeichnet als "Morphigeninsulfosäure nach Vahlen". Daß dies aber keine eindeutige Bezeichnung ist, geht aus dem oben Gesagten doch wohl zur Genüge hervor.

Entsprechend den verschiedenen Darstellungsweisen, waren auch die physiologischen Wirkungen meiner Morphigeninsulfurierungsprodukte nichts weniger als gleichartig.

Man kann die in meiner Arbeit mitgeteilten Tierversuche mit "Morphigeninsulfosäure" in 3 Gruppen teilen:

1. Experimente, in denen eine narkotische Wirkung beobachtet wurde, ohne eine gleichzeitige, mit blobem Auge wahrnebmbare Blutveränderung.

2. Solche, in denen eine narkotische Wirkung festgestellt wurde, die begleitet (aber wohl kaum verursacht) war von einer Veränderung des Blutstoffes.

3. Wird ein Versuch angeführt, in dem sich ein Sulfurierungsprodukt im Reagensglase als methämoglobinbildend erwies, gleichwohl aber weder eine narkotische noch überhaupt irgendeine Wirkung auf das lebende Tier besaß.

Diese Versuchsergebnisse rechtfertigten doch wohl meine Vermutung, daß durch Einwirkung von konzentrierter Schwefelsäure auf Morphigenin sowohl Produkte von narkotischer wie von methämoglobinbildender Wirkung entstanden. Daher war mein Bestreben darauf gerichtet, durch Abänderung des Sulfurierungsverfahrens eine Methode aufzufinden, durch die allein oder doch hauptsächlich die narkotischen Gifte gebildet wurden. Dies ist mir, wie ich selbst ausdrücklich mitteilte, nicht gelungen. Aber Pschorr ist dies innerhalb acht Monaten auch nicht geglüekt.

Ps ch orrs Vorwürfe gegen meine "Morphigeninsulfosäure" haben,

1) Nichtsdestoweniger ist in der Abhandlung von Pschorr und Bergell S. 31 zu lesen: „Die Unzulänglichkeit der von Vahlen aufgestellten Folgerungen wurde bereits zum Teil von dem einen von uns an anderer Stelle auf rein chemisch-analytischem Wege nachgewiesen!: 
wie schon gesagt, außer einer chemischen auch noch eine pharmakologische Seite, die freilich mit der Erledigung der ersteren gegenstandslos geworden ist.

Pschorr behauptete in seinem ersten Angriff: 1) meine "Morphigeninsulfosäure" sei weiter nichts als Phenanthrenchinonsulfosäure; 2. meine "Morphigeninsulfosäure" habe daher die Wirkung der Phenanthrenchinonsulfosäure, diese wirke aber" nur methämoglobinbildend, somit sei ich in meiner Beobachtung einer narkotischen Wirkung auf das gröbste getäuscht worden.

Jeder, der die kurze Angabe Pschorrs las, mußte glauben, er hätte eine Wirkung der "Morphigeninsulfosäure" auf das Blut erst entdeckt, die mir vollkommen entgangen wäre. Daß dem nicht so ist, brauche ich nicht noch einmal auseinanderzusetzen.

Ich glaubte nun, daß wenigstens diese Angabe Pschorrs, Phenanthrenchinonsulfosäure (fälschlich als „Morphigeninsulfosäure Vahlen" bezeichnet) wirke methämoglobinbildend, richtig sei. Es war nicht unmöglich, daß dadurch die Wirkung auf das Blut einiger meiner Morphigeninsulfurierungsprodukte ibre Erklärung gefunden hätte.

Nun findet sich aber in der Arbeit von Psehorr und Bergell als einziger experimenteller Beleg dafür, daß ihre „Morphigeninsulfosäure nach Vahlen" ein starkes Blutgift sei, nur ein Versuch an einem Hahn angefiihrt, in dessen Kammschnittblut Methämoglobin spektroskopisch nachgewiesen werden konnte. Man vergleiche dagegen einige meiner Versuche mit "Morphigeninsulfosäure", in denen das Blut des lebenden Tieres schon für das bloße Auge eine deutliche braune Farbe darbot.

Da nach allem, was ich gesagt habe, Pschorr und Bergells Tierversuche mit ihrer "Morphigensulfosäure nach Vahlen" gar keine Beziehung zu irgendwelchen Angaben von meiner Seite haben, so rerzichte ich auf ihre weitere Besprechung. Ich kann mir aber nicht versagen, daranf hinzuweisen, daß anch diese Versuche, $a b-$ gesehen von dem bereits erwähnten Bahn, nur an Mäusen, als wichtigsten Vertretern der "Warmblüter", angestellt worden sind.

Die Ergebnisse dieser Arbeit fasse ich in folgende Sätze zusammen:

1. Die von mir gegebene Beschreibung dei morphiumähnlichen Wirkung des. Epiosins wird durch neue Versuche auf das Unzweideutigste bestätigt.

2. Es ist Psehorr bisher nicht gelungen, irgendeine Angabe in meiner ersten Abhandlung, mag es sich um eine chemische Feststellung oder um eine Beobachtung an Tieren handeln, als unrichtig zul erweisen. 